University of Wollongong

Research Online

Faculty of Engineering and Information

Faculty of Engineering and Information

Sciences - Papers: Part A

Sciences

$1-1-2014$

Circular domain features based condition monitoring for low speed slewing bearing

Wahyu Caesarendra

University of Wollongong,wc026@uowmail.edu.au

Prabuono Buyung Kosasih

University of Wollongong, buyung@uow.edu.au

A Kiet Tieu

University of Wollongong, ktieu@uow.edu.au

Craig A. S Moodie

University of Wollongong, cam920@uow.edu.au

Follow this and additional works at: https://ro.uow.edu.au/eispapers

Part of the Engineering Commons, and the Science and Technology Studies Commons

Research Online is the open access institutional repository for the University of Wollongong. For further information contact the UOW Library: research-pubs@uow.edu.au 


\title{
Circular domain features based condition monitoring for low speed slewing bearing
}

\begin{abstract}
This paper presents a novel application of circular domain features calculation based condition monitoring method for low rotational speed slewing bearing. The method employs data reduction process using piecewise aggregate approximation (PAA) to detect frequency alteration in the bearing signal when the fault occurs. From the processed data, circular domain features such as circular mean, circular variance, circular skewness and circular kurtosis are calculated and monitored. It is shown that the slight changes of bearing condition during operation can be identified more clearly in circular domain analysis compared to time domain analysis and other advanced signal processing methods such as wavelet decomposition and empirical mode decomposition (EMD) allowing the engineer to better schedule the maintenance work. Four circular domain features were shown to consistently and clearly identify the onset (initiation) of fault from the peak feature value which is not clearly observable in time domain features. The application of the method is demonstrated with simulated data, laboratory slewing bearing data and industrial bearing data from Coal Bridge Reclaimer used in a local steel mill.
\end{abstract}

\section{Keywords}

slewing, domain, monitoring, speed, circular, low, features, bearing, condition

\section{Disciplines}

Engineering | Science and Technology Studies

\section{Publication Details}

Caesarendra, W., Kosasih, B., Tieu, A. Kiet. \& Moodie, C. A. S. (2014). Circular domain features based condition monitoring for low speed slewing bearing. Mechanical Systems and Signal Processing, 45 (1), 114-138. 
Manuscript for Mechanical Systems and Signal Processing

\title{
Circular Domain Features based Condition Monitoring for Low Speed Slewing Bearing
}

\author{
Wahyu Caesarendra*a,b, Buyung Kosasiha ${ }^{\mathrm{a}}$, Anh Kiet Tieu, ${ }^{\mathrm{a}}$, Craig A.S. Moodie $^{\mathrm{a}}$ \\ ${ }^{a}$ School of Mechanical, Materials and Mechatronic Engineering, University of Wollongong, Wollongong, New South \\ Wales 2522, Australia \\ ${ }^{b}$ Mechanical Engineering Department, Diponegoro University, Tembalang, Semarang 50275, Indonesia
}

\section{* Corresponding Author:}

Wahyu Caesarendra

School of Mechanical, Materials and Mechatronic Engineering,

University of Wollongong,

Wollongong, NSW 2522, Australia

Phone: 61 2+ 42213555

Fax: $612+42213477$

E-mail:wc026@uowmail.edu.au

Total number of pages: 48

Total number of figures: 20

Total number of tables: 7 


\title{
Circular Domain Features based Condition Monitoring for Low Speed Slewing Bearing
}

\begin{abstract}
This paper presents a novel application of circular domain features calculation based condition monitoring method for low rotational speed slewing bearing. The method employs data reduction process using piecewise aggregate approximation (PAA) to detect frequency alteration in the bearing signal when the fault occurs. From the processed data, circular domain features such as circular mean, circular variance, circular skewness and circular kurtosis are calculated and monitored. It is shown that the slight changes of bearing condition during operation can be identified more clearly in circular domain analysis compared to time domain analysis and other advanced signal processing methods such as wavelet decomposition and empirical mode decomposition (EMD) allowing the engineer to better schedule the maintenance work. Four circular domain features were shown to consistently and clearly identify the onset (initiation) of fault from the peak feature value which is not clearly observable in time domain features. The application of the method is demonstrated with simulated data, laboratory slewing bearing data and industrial bearing data from coal bridge reclaimer used in a local steel mill.
\end{abstract}

Keywords: Circular domain features; condition monitoring; low speed slewing bearing; piecewise aggregate approximation.

\section{Nomenclature}

\begin{tabular}{cl}
\hline$C$ & Number of occurrence \\
$d_{m}$ & Mean bearing diameter \\
$d_{r}$ & Diameter of rolling element \\
EMD & Empirical mode decomposition \\
FFT & Fast Fourier transform \\
$f s$ & Sampling frequency \\
IMF & Intrinsic mode function \\
$i^{t h}$ & Occurrence data point \\
$I R_{r p m}$ & Rotational speed of inner bearing ring \\
$O R_{r p m}$ & Rotational speed of outer bearing ring \\
$k$ & Circular kurtosis [11]
\end{tabular}




$\begin{array}{cl}m & \text { Circular skewness [11] } \\ N & \text { Number of data points of vibration signal } \\ n & \text { Length of reduced-data, } n=\frac{N}{w} \\ \mathrm{~A}_{\mathrm{RMS}} & \text { RMS of white noise amplitude } \\ r & \text { Radius of circular plane } \\ R & \text { Resultant vector length } \\ S & \text { Number of samples for a half sinusoidal signal } \\ \mathbf{t} & \text { Time vector, } \mathbf{t}=\left(t_{1} \cdot t_{2}, \ldots, t_{N}\right) \\ t_{\mathrm{max}} & \text { Total time, } t_{\mathrm{max}}=t_{N} \\ V & \text { Circular variance } \\ \mathrm{V}_{\mathrm{RMS}} & \text { RMS of vibration signal amplitude } \\ w & \text { Window size of PAA } \\ \mathbf{x} & \text { Reduced data, } \mathbf{x}=\left(x_{1} . x_{2}, \ldots, x_{n}\right) \\ \mathbf{y} & \text { Sampled vibration data, } \mathbf{y}=\left(y_{1}, y_{2}, \ldots, y_{N}\right) \\ Z & \text { Number of rolling elements } \\ Z_{i} & \text { Circular plane of angular domain } \alpha_{i} \\ \bar{Z} & \text { Circular mean } \\ \boldsymbol{\alpha} & \text { The occurrence data set in circular domain, } \boldsymbol{\alpha}=\left(\alpha_{1}, \alpha_{2}, \ldots, \alpha_{I}\right) \\ \beta & \text { Reversible angle of slewing bearing } \\ \phi & \text { Shifting factor } \\ \lambda & \text { Frequency that triggers the change of ellipsoid orientation }\end{array}$

\section{Introduction}

Slewing bearing is a subgroup of rolling element bearing commonly used in large industrial machineries such as turntable, steel mill cranes, offshore cranes, rotatable trolley, excavators, reclaimers, stackers, swing shovels, and ladle cars. They typically support high axial and high radial load. Slewing bearings are often critical production part. An unplanned downtime when a bearing breaks down can be very expensive due to the loss of production. Moreover as replacement of large slewing bearing can take several months to arrive due to long manufacturing and delivery time, plants often carry spare bearing to guard against these unforeseen circumstances adding an extra cost. In order to prevent unplanned downtime, a condition monitoring and prognosis method is needed.

Although there is no existing standard criterion for speed classification of rotating machinery, some published literatures mentioned that the rotating speed below than $600 \mathrm{rpm}$ is categorized as low rotating speed machinery [1-3]. According to ISO 2372 which mentions that the classifications 
of vibration velocity severity covers machines with rotational speeds ranging from 600 to $1200 \mathrm{rpm}$ and [4] mentioned that speed greater than $600 \mathrm{rpm}$ is high speed machinery, thus in this paper the rolling bearing run at speed greater than $600 \mathrm{rpm}$ is considered typical rolling bearing.

There have been extensive study of vibration analyses and features extractions for condition monitoring, fault diagnosis and prognosis of typical rolling bearing [5-8] and the results shown these techniques can effectively monitor the changes of bearing condition. In typical rolling bearing, once a fault is initiated the bearing can deteriorate rapidly within few hundreds/thousands revolutions and result in changes of vibration within very short time from the onset of the fault [8]. Thus in this case the use of features extraction methods such as time domain and frequency domain features calculation is effective to distinguish the bearing condition. However, the methods and features suitable for one directional typical rotating bearing cannot be applied effectively for identifying the abnormal condition of low rotational speed bearing [2] especially in extremely low rotational speed $(\approx 1 \mathrm{rpm})$ slewing bearing [9]. This is due to the low impact energy emission from the rotating elements contact with a defect spot might not show an obvious change in vibration signature correspond to the bearing damage condition and thus become hardly detectable with conventional vibration analysis [10]. Moreover the bearing signal is also deeply masked by the background noise. Therefore, although time domain features [5-8] are extracted from the signal where the noise is dominant, the onset of bearing fault is still undetectable [9]. Eventually the amplitude is greater than the background noise, but by that stage features value will have increased substantially signifying that significant change of bearing condition has already occurred. Often by this stage the bearing condition is already close to unsustainable operation or near to failure.

To overcome the problem and prevent the sudden breakdown from occurring, alternative features which are able to identify the incipient fault is needed. This paper presents a novel application of circular domain features calculation based condition monitoring method for low rotational speed slewing bearing. In contrast to the previous angle and cyclic domain analysis discussed in $§ 2$, this paper employed circular analysis to extract the circular domain features. Circular analysis is a subclass of statistic where it is different to general time domain statistical analysis. In circular analysis the statistical features such as mean, variance, skewness and kurtosis are calculated from the data distributed in circular domain or angular domain. Circular features were initially introduced in biological and medical science fields [11-13]. This paper combines piecewise aggregate approximation (PAA) data reduction process and circular features calculation (inspired by Berens [13]) as the monitored variables. The paper demonstrates the efficiency of the proposed method in detecting the onset of slewing reversible bearing fault. Reversible slewing bearing alternately 
rotates in clockwise rotation and anti-clockwise directions. The general steps of the proposed method are illustrated in Fig. 1. The method consist of three main steps: (i) reduction of the vibration data using PAA process and construction of neighborhood correlation plot of the reduced data, (ii) determination of the shape of the neighborhood plot using ellipse least-square fitting for pattern classification and (iii) plot the distribution of the ellipse shape in angular domain, and calculation of the circular domain features (in the paper, bearing data between February to August 2007 was used as the test case). The detail description of Fig. 1 especially the signals output between the boxes from original vibration signal to ellipsoid pattern classification is presented in §4.6. The proposed method is compared to time domain features and advanced signal processing methods such as wavelet decomposition and empirical mode decomposition (EMD).

The paper is organized as follow: §2 reviews angular resampling method’s application in rotating machinery; \$3 presents the comparable advanced signal processing methods: the wavelet transform and the EMD method combined with statistical features; §4 discusses the theory and the merits of PAA method as slewing bearing signal processing tool, verification of PAA to identify frequency changes using simulated data, the application of PAA on vibration data, direct ellipse least-square fit classification, monitoring of faulty slewing bearing condition using PAA process, and detail signal processing flow process from original slewing bearing vibration signal to ellipsoid pattern classification; \$5 presents the circular feature analysis including circular domain transformation, circular features calculation, and application of circular features on laboratory slewing bearing based PAA result; and $\S 6$ presents the discussion and the conclusions of the works.

Fig. 1 Diagram of circular domain features extraction method.

\section{Angular Resampling}

Angular resampling is a useful signal processing methodology in vibration analysis which has been proven to work well in rotational machineries with variational speed. Ref. [14] is the first known attempt to use angular resampling by means of angle domain analysis. The paper demonstrated the different results obtained from simulated sinusoidal signal sampled at time domain and angular domain. Moreover, the sinusoidal signal is simulated with impulse signal which represent the bearing fault and varying speed using mathematical function. It is shown that the simulated fault is clearly identified in angular domain analysis even though the speed changes. Many researchers have used angular resampling to perform order domain analysis or order tracking method [15, 16]. Unlike frequency domain analysis which reveals the dominant frequency content 
of the vibration signal, the order domain analysis or order tracking method calculates multiples running speed content of the variable shaft rotation. Fyfe et al. [15] stated that speed-related vibrations can be identified easily using order tracking method. The authors presented the two existing order tracking methods namely: conventional order tracking and computed order tracking. The conventional order tracking employed special instrumentations such as ratio synthesizer and anti-aliasing tracking filter to sample directly the analog vibration signal at constant increment angle (i.e. $\Delta \theta$ ). Computed order tracking method resamples the acquired discrete vibration data sampled at uniform $\Delta t$ into a constant angular increments, $\Delta \theta$. Once a specified block of data sampled at constant $\Delta \theta$ (angle domain samples) from both methods has been obtained, the order spectrum is computed using Fast Fourier Transform (FFT). Moreover, the effect of various factors on the accuracy of the computed order tracking method was investigated. However, the data used for computing the order tracking is the simulation data generated from run-up simulation model instead of actual data. An extended work of the two previous order tracking methods described in [15] was proposed by Bosssley et al. [16]. The focus of these works was in the assessment of the accuracy of the three different order tracking methods: conventional order tracking, computed order tracking and hybrid of the two. The methods were applied in simulation vibration signals produced from a power station gas turbine shaft.

Case studies where angular resampling has been applied include rolling element bearing $[17,18]$, gearbox [19-21], wind turbine [22, 23] and induction motor [24] analyses. The angular resampling application in [19] used the acceleration signals directly without the need for an encoder signal. The resampling algorithms developed in the above published literatures [19-21] are applicable for small speed fluctuation cases. Ref. [22] offered an improved angular resampling algorithm for variable speed machineries such as wind turbine. A recent and promising method for natural roller bearing fault detection based on instantaneous angular speed measurement has been presented by Renaudin et al. [18]. The instantaneous angular speed measurement with a true angular sampling is carried out using magnetic and optical encoders. The instantaneous angular speed measures the kinematics of the device using the principle of pulsed timing method. This study proved that instantaneous angular speed exhibits small periodic fluctuations in the angular frequency domain when spalled damage has occurred.

The application of angular resampling in 'slewing bearing' has been considered in 1990 [25] as an internal steel metal making company report. However, the report only presented the theoretical analysis without any example in real case. To date, there are limited literatures that discuss the application of angular resampling in slewing bearing case. 


\section{Comparable Advanced Signal Processing Methods: Wavelet Transform and Empirical Mode Decomposition (EMD)}

Yang and Widodo [26] defined signal processing as a method which when applied on raw vibration signal enhance the signal's reliability and improve the accuracy of the subsequent signal processing or signal analysis. In dealing with vibration signal with non-stationary characteristics, the wellknown advanced techniques for signal processing are time-frequency technique (short-time Fourier transform) and time-scale technique (wavelet transform). Other powerful signal processing methods for non-stationary signals are Wigner-Ville distribution (WVD) and empirical mode decomposition (EMD). In this paper, wavelet transform and EMD were selected as the comparable signal processing methods to be compared to the proposed method.

\section{Wavelet Transform}

Wavelet transform decomposes non-stationary input signal into a linear combination of a timescale unit. It decomposes original signal and organizes it into several signal components according to the translation of the mother wavelet (or wavelet basis function), which changes the scale and shows the transition of each frequency component [27]. In this paper, multilevel one-dimensional wavelet decomposition is utilized. Previous study employed wavelet decomposition for fault diagnosis of induction motor using transient stator current signal [28]. A review of wavelet transform has been presented by Feng et al. [29]. In wavelet decomposition, the input signal is basically decomposed into two coefficients. The input signal that passes through the low-pass filter becomes the 'approximation coefficient' and the input signal which passes through the high-pass filter becomes the 'detail coefficient.' Since the low-frequency content is the most important part, the 'approximate coefficient' is passed to the next wavelet decomposition loop in multilevel wavelet decomposition process. Fig. 2 shows wavelet decomposition structure illustrating the decomposition of slewing bearing vibration signal into approximate coefficient (A) and detail coefficient (D) at each level. In this study, up to three level decompositions were carried out. Features such as mean, variance, skewness and kurtosis were calculated from the detail coefficients of level 3 (D3) and the results are plotted in Fig. 17 as a comparison with the proposed method.

Fig. 2 Three level wavelet decompositions of the slewing bearing data (acquired on May $25^{\text {th }}$ ). 
EMD [30] has been shown to be adaptable in applications where the signal is non-stationary (e.g. Braun and Feldman [31]). A recent review of EMD applications in fault diagnosis of rotating machinery can be found in Ref. [32]. The authors presented the detail of EMD in various applications e.g. bearings, gears, and rotors etc. The authors also reviewed existing EMD methodologies and classified it into three different groups namely: (1) original EMD method alone, (2) improved EMD methods, and (3) combinations of EMD with other methods such as artificial neural network and support vector machine. In this paper, original EMD [33] is used for nonstationary slewing bearing data. The main function of EMD is to decompose the original vibration signal into several signals which has specific frequency called intrinsic mode functions (IMFs) based on the enveloping technique. The results of IMFs are from high frequencies to low frequencies.

In condition monitoring of rolling bearing, EMD is used to reveal the frequency content of vibration signal by decomposing the original signal into several IMFs in order to determine whether the bearing signal has specific frequency content corresponds to the bearing fault frequencies or not. A bearing fault such as outer race, inner race or rolling element fault has occurred when one of the IMF frequencies is identical to one of the bearing fault frequencies (for example as shown in Table 1) [34].

Similar to the proposed method and two previous comparison methods, EMD is used in slewing bearing signal to identify the onset of bearing fault. The selected EMD results of slewing bearing data acquired on March 1, May 25 and August 30 are presented in Table 2, 3 and 4, respectively. Since the low frequencies components are of utmost important in low speed slewing bearing, the summation of these low IMF frequencies is used as the input signal in the features calculation step (Fig. 3). The resulting features calculation is then plotted in Fig. 17 as additional comparison.

Table 1 Fault frequencies of slewing bearing (run at $1 \mathrm{rpm}$ and $4.5 \mathrm{rpm}$ for slewing bearing test-rig and slewing bearing Bridge Reclaimer, respectively)

Table 2 EMD result of vibration data on March $1^{\text {st }} 2007$.

Table 3 EMD result of vibration data on May 25 2007.

Table 4 EMD result of vibration data on August 30 2007.

Fig. 3 EMD results of slewing bearing data (vibration data on May $25^{\text {th }}$ ). 


\section{Piecewise Aggregate Approximation (PAA)}

\subsection{Theory}

PAA data reduction process was introduced by $\mathrm{Yi}$ and Faloutsos [35] and Keogh et al. [36] independently. It was first developed as a data reduction technique for large time series data whilst keeping its characteristic. To reduce the data length, one sequence of sampled vibration data, $\mathbf{y}=\left(y_{1}, y_{2}, \ldots, y_{N}\right)$ with $N$ number of data points is divided into $w$ window of equal size termed 'frames'. The mean value of the data in each frame and the vector of these mean values becomes the reduced data representation or PAA result. The mean value of data in one frame is given as follow:

$$
x_{n}=\frac{1}{w} \sum_{j=w(n-1)+1}^{w n} y_{j}
$$

The PAA result of Eq. (1) is the vector $\mathbf{x}=\left(x_{1}, x_{2}, \ldots, x_{n}\right)$. One cycle of sinusoidal signal and the reduced data representation are shown in Fig. 4. In the next step, the PAA result is used as processed data to identify the frequency alteration.

Fig. 4 One cycle of sinusoidal signal $(1 \mathrm{~Hz})$ sampled with $64 \mathrm{~Hz}$ sampling frequency is shown in solid blue curve. The data is divided into 8 frames (solid red line). The calculated mean value of sampled data in each frame is shown in yellow square shape. All mean values in the vector of $\mathbf{x}=$ $\left(x_{1}, x_{2}, \ldots, x_{8}\right)$ is the PAA result. The index $w 1, w 2, \ldots w 8$ is the equal window size or "frame".

The saved vibration data obtained from extremely low rotating speed bearing ( $\approx 1 \mathrm{rpm}$ ) acquired at certain sampling rate over one minute or more requires the data reduction process. In this study, PAA data processing is used to reduce the saved data but still can extract the high frequency component from short-duration signal (1 second) making it suitable in the present analysis where slewing bearing signal were sampled few times over long-duration each day during the months long experiment duration. The high frequency signal indicates the presence of fault initiation. The more high frequency component identified in one data set indicates that the bearing condition deteriorates further.

In addition, when slewing bearing condition is normal, the bearing vibration signal amplitude is much lower than the background noise amplitude. As bearing condition deteriorates and the onset 
of damage has occurred, the bearing signal amplitude will increase although may still be below than the background noise amplitude. Therefore, if time-domain features are calculated from this signal, the feature values are still indistinguishable to normal condition bearing feature values. When bearing condition is close to unsustainable operation, the bearing signal become stronger and after some time the signal will be greater than the background noise. At this stage the time-domain features will be more sensitive. With PAA method combined with circular-domain features calculation, the onset of damage can be identified allowing preventive maintenance to be planned.

\subsection{Identification of Frequency Alteration from the Shift of the Ellipsoid Orientation}

The question regarding the application of PAA is how frequency alteration is detected. This is very much dependent on the window size used. The investigation of the effect of different window size is carried out using simulated data. Two different signals $(1 \mathrm{~Hz}$ and $5 \mathrm{~Hz}$ frequency) sampled with sampling frequency of $64 \mathrm{~Hz}$ are shown in Figs. 5 to 7 . Signals with amplitude 1 are simulated in one second. Each signal is reduced with equal window size. Three different window sizes namely 2, 4 and 8 are used in Figs. 5, 6 and 7 respectively. The reduced data or PAA result, $\mathbf{x}=\left(x_{1}, x_{2}, \ldots\right.$, $\left.x_{n}\right)$ is plotted in the form of neighborhood correlation plot, $x(n+1)$ against $x(n)$. For $1 \mathrm{~Hz}$ signal, the neighborhood correlation plots show the ellipsoid axis is oriented at 45 degree angle as depicted in Figs. 5(a) to 7(a) for window size of 2, 4 and 8 . While, in case of $5 \mathrm{~Hz}$ signal, the ellipsoid still inclines at 45 degree angle with window size of 2 but changes to 135 degree angle when window sizes 4 and 8 are used.

Moreover, the effect of different frequency with fixed window size is investigated with four different frequencies namely $1 \mathrm{~Hz}, 2 \mathrm{~Hz}, 3 \mathrm{~Hz}$ and $6 \mathrm{~Hz}$ sampled at $64 \mathrm{~Hz}$. A window size of 4 is employed for each signal. It can be seen in Fig. 8 in the neighborhood correlation plots, the ellipsoid of frequency $1 \mathrm{~Hz}, 2 \mathrm{~Hz}$, and $3 \mathrm{~Hz}$ is oriented at 45 degree angle (right ellipsoid pattern). But, it is shifted to 135 degree (left ellipsoid pattern) when the frequency is $6 \mathrm{~Hz}$. This implies that frequency alterations can change the angle of the ellipsoid axis of the reduced data obtained by PAA process.

Fig. 5 Neighborhood correlation plots of $1 \mathrm{~Hz}$ and $5 \mathrm{~Hz}$ sinusoidal signals with window size $=2$.

Fig. 6 Neighborhood correlation plots of $1 \mathrm{~Hz}$ and $5 \mathrm{~Hz}$ sinusoidal signals with window size $=4$.

Fig. 7 Neighborhood correlation plots of $1 \mathrm{~Hz}$ and $5 \mathrm{~Hz}$ sinusoidal signals with window size $=8$.

Fig. 8 Sinusoidal signal and PAA process (left) and neighborhood correlation plot based PAA result (right) for different frequencies with fix window size of 4: (a) $1 \mathrm{~Hz}$; (b) $2 \mathrm{~Hz}$; (c) $3 \mathrm{~Hz}$; (d) $6 \mathrm{~Hz}$. 


\subsection{Application in Real Case}

Slewing bearing data from two different sources were used as the test case data.

\section{Lab Slewing Bearing Rig}

The first data used in this paper was acquired from slewing bearing test-rig. The test-rig can be operated at 1 to $12 \mathrm{rpm}$. The test-rig was designed to simulate the real working conditions of a steel making company. There are four real working conditions considered in the design of the test-rig: (1) The test-rig is designed to operate between 1 to $12 \mathrm{rpm}$ rotational speed typical of operational speed in steel industry. (2) It must be able to operate with reversible rotational direction. (3) The load applied into the bearing is high load. (4) The test-rig was located in open air which possible to contaminate from dust environment e.g. coal dust. In this paper, the test-rig was operated at $1 \mathrm{rpm}$. The slewing bearing used was an axial/radial bearing supplied by Schaeffler (INA YRT260) with an inner and outer diameter of $260 \mathrm{~mm}$ and $385 \mathrm{~mm}$. The vibration data were acquired from four accelerometer sensors installed on the inner radial surface at 90 degree to each other. The accelerometers were IMI608 A11 ICP type sensor. The accelerometers were connected to high speed Pico scope DAQ (PS3424). The vibration data was collected on daily basis with $4880 \mathrm{~Hz}$ sampling rates during the period between February to August 2007 (138 days). In order to accelerate the bearing defect, coal dust was injected into the bearing in the middle of April 2007 (58 days after the test started). The schematic of the slewing bearing test rig showing the main drive gear reducer, the hydraulic load and how the bearing is attached is presented in Fig. 9(a); and the slewing bearing and sensor placement is shown in Fig. 9(b).

Fig. 9 (a) Schematic of lab slewing bearing rig; (b) Slewing bearing and sensors placement.

\section{Coal Bridge Reclaimer}

Industrial bearing data used in this paper was acquired from slewing bearing used in a Coal Bridge Reclaimer. The bearing usually rotates at approximately 4.5rpm. Type of slewing bearing used is Rothe Erde 4.3m diameter. The accelerometers employed were IMI512, 500mV/g ICP type piezos. Data was collected from 2003 to 2006 at each sample point approximately once per month and was captured via an industrialized portable DAQ unit (NI 5102).

\section{PAA Application in Lab Slewing Bearing Rig and Coal Bridge Reclaimer Data}

PAA data reduction process with $w=32$ and 16 were used in processing the lab slewing bearing data and the reclaimer data. The result is plotted in neighborhood correlation plot, $x(n+1)$ against 
$x(n)$ as shown in Fig. 10. Three different neighborhood plot orientations are clearly visible namely (1) centered; (2) left shifted at 135 degree; and (3) right shifted at 45 degree. When the bearing is in good condition the neighborhood plot appears to have centered orientation. When the condition starts to deteriorate the ellipsoid is oriented to 135 degree (left shifted); and when the condition of bearing is close to failure the neighborhood plot is at 45 degree (right shifted). The question is 'what triggers the change of the orientation?' The answer is the change of frequency explained in subsection 4.2. Furthermore, the data scattered in neighborhood plot with centered orientation is due to the background noise. The background noise is the common issue in practice. When the acquired signal contains dominant background noise, the neighborhood plot produce centered orientation. Because the low amplitude of bearing signal is associated with good bearing condition, when the bearing is in good condition the bearing signal is very weak and deeply masked by the background noise.

To demonstrate the effect of the change of bearing signal magnitude, another simulated signal is produced as shown in Fig. 11. The $5 \mathrm{~Hz}$ frequency signal is sampled with sampling frequency identical to the sampling frequency of lab slewing bearing rig data i.e. $4880 \mathrm{~Hz}$ for 2 sec. The first one second signal contains the sinusoidal signal plus white noise where the amplitude of sinusoidal signal is greater than the amplitude of white noise shown with blue signal, and the last one second simulates the weak $5 \mathrm{~Hz}$ signal deeply buried in white noise (Fig. 11(a)) shown in red. Window size of 40 was used for each type of signal resulting in 122 segments. Plot of the 122 reduced data in the form of neighborhood correlations plot, $x(n+1)$ against $x(n)$. The data is scattered in right ellipsoid orientation as shown in the left plot in Fig. 11(b).

When the amplitude of background noise is larger than the bearing signal, the data is scattered in circular formation. This demonstration illustrates the application of neighborhood correlation plot for bearing signal detection. An amplitude ratio denoted Fig. 9 was computed by $V_{\text {RMS }} / A_{R M S}$, where $\mathrm{V}_{\mathrm{RMS}}$ is the root mean square of vibration signal amplitude and $\mathrm{N}_{\mathrm{RMS}}$ is the root mean square of white noise amplitude. The green color signal is the PAA reduced data.

\subsection{Ellipse Least-Square Fitting Method for PAA Results}

The right or left ellipsoid oriented patterns presented in Figs. 5-8 only occur in pure sinusoidal signal. In practice, the PAA result or data reduced representation is scattered in neighborhood correlation plots as shown in Fig. 10. Even though the PPA result is scattered but it still has ellipsoid outline. Thus, to establish the ellipsoid outline curve from the scattered data, direct least- 
square ellipse fitting method [37] is used. The method [37] has high computational efficiency compared to conventional ellipse fitting method [38, 39]. The fitting equation is given by a second order polynomial conic equation given below

$$
F(\mathbf{p}, \mathbf{s})=a x^{2}+b x y+c y^{2}+d x+e y+f=0
$$

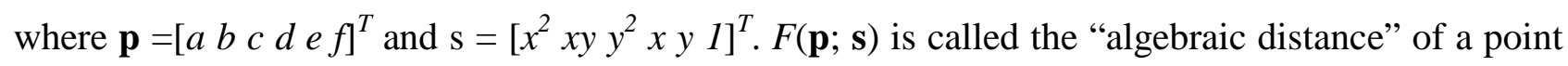
$(x, y)$ to the conic $F(\mathbf{p} ; \mathbf{s})=0$. The fitting of a general conic is done by minimizing the sum of the squared algebraic distances of the curve to the $n$ data points $\mathbf{s}_{i}[40]$.

$$
\Delta_{A}(\mathbf{p})=\sum_{i=1}^{n} F\left(\mathbf{s}_{i}\right)^{2}
$$

In many articles on ellipse fitting method, the different is only in the constraint applied to the parameter vector $\mathbf{p}$ in order to avoid the trivial solution $\mathbf{p}=0_{6}$ (e.g Refs. [38, 39]). These constraints are either linear or quadratic. If the constraint set on the parameter vector is quadratic, the minimization of Eq. (3) can be solved using one of least-square methods such as Tikhonov regularization by considering rank-deficient generalized eigenvalue system [41]:

$$
\mathbf{D}^{T} \mathbf{D p}=\lambda \mathbf{C p}
$$

where $\mathbf{D}=\left[\mathbf{s}_{1}, \mathbf{s}_{2}, \ldots, \mathbf{s}_{n}\right]^{\mathrm{T}}$ is called the design matrix and $\mathbf{C}$ is the matrix that expresses the constraint. In direct ellipse-specific fitting method [37], the quadratic constraint of $4 a c-b^{2}=1$ is employed. This constraint can be expressed in the matrix form $\mathbf{p}^{T} \mathbf{C} \mathbf{p}=1$ as

$$
\mathbf{p}^{T}\left[\begin{array}{rrrrrr}
0 & 0 & 2 & 0 & 0 & 0 \\
0 & -1 & 0 & 0 & 0 & 0 \\
2 & 0 & 0 & 0 & 0 & 0 \\
0 & 0 & 0 & 0 & 0 & 0 \\
0 & 0 & 0 & 0 & 0 & 0 \\
0 & 0 & 0 & 0 & 0 & 0
\end{array}\right] \mathbf{p}=1
$$

By minimizing $E=\|\mathbf{D} \mathbf{p}\|^{2}$ subject to the constraint $\mathbf{p}^{T} \mathbf{C} \mathbf{p}=1$ and introducing the Lagrange multiplier $\lambda$ and differentiating [37], the following quadratic equation is obtained 


$$
\begin{gathered}
2 \mathbf{D}^{T} \mathbf{D} \mathbf{p}-2 \lambda \mathbf{C p}=0 \\
\mathbf{p}^{T} \mathbf{C} \mathbf{p}=1
\end{gathered}
$$

This may be rewritten as

$$
\begin{gathered}
\mathrm{Sp}=\lambda \mathbf{C p} \\
\mathbf{p}^{T} \mathbf{C p}=1
\end{gathered}
$$

where $\mathbf{S}$ is the scatter matrix $\mathbf{D}^{T} \mathbf{D}$. This system can be solved by considering the generalized eigenvectors of (7). If $\left(\lambda_{i}, \mathbf{u}_{i}\right)$ solves (7), then so does $\left(\lambda_{i}, \mu \mathbf{u}_{i}\right)$ for any $\mu$ and thus from (8) we can find the value of $\mu_{\mathrm{i}}$ as $\mu_{i}^{2} \mathbf{u}_{i}^{T} \mathbf{C} \mathbf{u}_{i}=1$ as define

$$
\mu_{i}=\sqrt{\frac{1}{\mathbf{u}_{i}^{T} \mathbf{C} \mathbf{u}_{i}}}=\sqrt{\frac{1}{\mathbf{u}_{i}^{T} \mathbf{S} \mathbf{u}_{i}}}
$$

Finally, setting $\hat{\mathbf{p}}_{i}=\mu_{i} \mathbf{u}_{i}$ solves (6).

We note that the solution of the eigensystem (7) yields six eigenvalue-eigenvector pairs $\left(\lambda_{\mathrm{i}}, \mathbf{u}_{\mathrm{i}}\right)$. Each of these pairs $\left(\lambda_{\mathrm{i}}, \mathbf{u}_{\mathrm{i}}\right)$ gives rise to a local minimum if the term under the square root of (9) is positive. $\mathbf{S}$ is positive definite, so the denominator $\mathbf{u}_{i}^{T} \mathbf{S} \mathbf{u}_{i}$ is positive for all $\mathbf{u}_{i}$. Therefore, the square root exists if $\lambda_{\mathrm{i}}>0$, so any solutions to (6) must have positive generalized eigenvalues.

From the fitted ellipse three possible ellipsoid orientations are obtained labelled as ' 0 ' for centred orientation, ' 1 ' for right shifted orientation (45 degree) and ' 2 ' for left shifted orientation (135 degree). This method is used as the ellipsoid classification recognition in the circular feature analysis.

Fig. 10 Results of PAA data reduction of the lab test data: (a) March 2007; (b) May 2007; (c) August 2007; and results of Coal Bridge Reclaimer data: (d) May 2004; (e) January 2005; (f) September 2006.

Fig. 11 (a) Simulated 5Hz signal plus white noise with different amplitude ratio; (b) PAA result or data reduced representation plotted in neighborhood correlation plot. 


\subsection{Faulty Slewing Bearing Condition Monitoring Using PAA Process}

Typical progressive deteriorating condition of slewing bearing can be viewed as composed of four stages shown in Fig. 12. During stage I the bearing is still in good condition and the amplitude of the background noise is larger than the amplitude of the bearing signal. In this stage, there is no fault in the bearing and the weak bearing signal is deeply buried in the background noise. Using a window size of 40 , the neighborhood correlation plot shows centered or sphere orientation. Stage II is 'defect inception stage'. In this stage, the onset of damage starts to occur as signified by the increased amplitude of bearing signal. This stage usually does not last long. The neighborhood correlation plot of this stage has evolved from sphere into a right ellipsoid orientation (45 degree). In stage III, the progressive fault occurs indicated by the presence of unknown high frequency signal. The neighborhood correlation plot ellipsoid orientation is switched over from right to left. In slewing bearing case, this can occur when bearing signal frequency increase suddenly after faults initiation. As the defects strike another surface it excites the resonance frequency of sensor, bearing or test-rig itself [42] and produces the typical frequency signals which are higher than bearing fault signals. In the last stage, the bearing is in severely damaged condition signified by high bearing fault signal amplitude. At this stage, the ellipsoid has right orientation again. The simulation demonstrates that any change of bearing condition will manifest in change of ellipsoid orientation.

The question now is what if the orientation shift is an isolated instance? Can it still be reliably used to monitor the progressing fault? To get definitive detection of bearing condition the ellipsoid orientation is considered as bearing signal feature and presented in circular domain.

Fig. 12 Progression of simulated bearing signal degradation and the detection of fault occurrence using PAA. This signal is simulated with sampling frequency of 4880 and PAA window size of 40. Stage I: $5 \mathrm{~Hz}$ signal + white noise with amplitude ratio is 0.1 . Stage II: $5 \mathrm{~Hz}$ signal + white noise with amplitude ratio of 0.4 . Stage III: $55 \mathrm{~Hz}$ signal + white noise with amplitude ratio of 0.4 . Stage IV: $5 \mathrm{~Hz}$ signal + white noise with amplitude ratio of 1 . The four neighborhood plots show the evolution of the ellipsoid and its orientation change.

\subsection{Signal Processing Flow Process from Original Vibration Signal to Ellipsoid Pattern Classification}


The illustration of signal processing based on PAA and ellipse least-square fitting method for slewing bearing signal acquired on May $25^{\text {th }}$ is shown in Fig. 13. Each 1 second data set is examined instead of whole data set in 30 seconds to extract the high frequency component in short duration. Using the neighborhood correlation plot combined with ellipse fitting method, this high frequency component can be identified. The three different data at 4 second, 11 second, and 23 second were selected for illustration. As shown in Fig. 13, each second of data set which contains 4880 samples was reduced using PAA method with window size of 8 . The PAA result becomes data reduced representation with 610 samples. Then, the data reduced are plotted in neighborhood correlation plot, $x(n+1)$ against $x(n)$. Using ellipse least-square fitting method [16] explained in \$4.4, the shape of neighborhood correlation plot can be identified and classified into three different orientations: 'left shifted ellipsoid', 'right shifted ellipsoid', and 'centered'. Since the onset of bearing fault is indicated by the presence of high frequency signal component and represented by the right shifted ellipsoid, thus the right shifted ellipsoid is recorded as the term of 'occurrence' and is used in the next step of circular domain transformation (Fig. 11) and circular domain features calculation (Figs. 12 and 13).

Fig. 13 Illustration of signal processing based on PAA method and ellipse least-square fitting method using vibration data acquired on May $25^{\text {th }}$ (day 90 ).

\section{Circular Features Analysis}

\subsection{Circular Domain Transformation}

The working principle of circular features calculation is different to time domain features calculation. In time domain features calculation, the features are calculated directly from vibration signal. The time domain features calculated from normal bearing signal are different to faulty bearing signal. This condition has been recognized in typical speed bearing (>600 rpm). In slewing bearing case, the bearing fault signal is weak and background noise is dominant, thus time domain features become undetectable to the change of bearing condition especially the onset of bearing fault due to features are calculated in noisy signal. This drawback is addressed in this study by introducing an alternative method called circular domain features calculation. 
To calculate features in circular domain the processed data using PAA should be transformed first into angular domain. The purpose is to identify the changes of bearing condition, particularly to identify the presence of high frequency signal component due to the contact between rolling element and defect spot within a definite sampling period e.g. one second ( $\approx 6$ degree of rotation) for bearing run at $1 \mathrm{rpm}$. In practice, the slewing bearing rotates in reversible mode at predefined span $\beta$ e.g. $360^{\circ}, 180^{\circ}, 120^{\circ}$ or $90^{\circ}$. Therefore the time domain when the vibration data is collected and converted initially to an angular scale in degree by

$$
\boldsymbol{\theta}=\left(\frac{\mathbf{t}}{t_{\max }}\right) \cdot \beta
$$

where $\theta$ is the angular scale vector in degree, $\mathbf{t}=\left(t_{1} \cdot t_{2}, \ldots, t_{N}\right)$ is time occurrence (the member of vector $\mathbf{t}$ is depends on the time occurrence of each 1 second $\left(\approx 6^{\circ}\right)$ analysing using PAA method), and $t_{\max }=t_{N}$ is the total time the bearing rotate in one direction. If the bearing is run at $1 \mathrm{rpm}$ with $\beta=180^{\circ}$, the duration of slewing bearing to complete one angular span, $t_{\max }$ is 30 second. It should be noted that the interval $\Delta t$ is corresponds to the sampling frequency, $f s$ of the vibration data. Then from the angular scale in degree, it is converted to an angular scale in radians by

$$
\alpha=\left(\frac{\boldsymbol{\theta} \cdot \pi}{180}\right)
$$

where $\alpha$ is the angular scale in radians. Alternative straight forward method to transform time vector, $\mathbf{t}=\left(t_{1} . t_{2}, \ldots, t_{N}\right)$ into angular direction, $\boldsymbol{\alpha}=\left(\alpha_{1}, \alpha_{2}, . ., \alpha_{N}\right)$ is

$$
\boldsymbol{\alpha}=\left(\frac{\mathbf{t}}{t_{\max }}\right) \cdot\left(\frac{\beta}{360}\right) \cdot 2 \pi
$$

Noted that Eq. (12) computes the entire linear vector (time domain) and converted into angular vector (circular domain). In this paper, $\alpha$ is calculated according to PAA result (each sample of occurrence). Therefore, the new vector of $\alpha$ is $\boldsymbol{\alpha}=\left(\alpha_{1}, \alpha_{2}, \ldots, \alpha_{C}\right)$ where $C$ is number of occurrences and each data point of occurrence $\alpha_{1}, \alpha_{2}, . ., \alpha_{i}$ is denoted as $i$. 
The PAA method as frequency alteration identification and circular domain transformation is illustrated in Fig. 14. One second $\left(\approx 6^{\circ}\right)$ of time series vibration (May $25^{\text {th }} 2007$ ) containing 4880 sampled points is used as an input for PAA method. The occurrence and non-occurrence is identified as " 1 " and “ 0 " respectively. The distribution of circular occurrence " 1 " in circulardomain is shown. Subsequently circular features such as circular mean, circular variance, circular skewness and circular kurtosis are calculated to see the statistical behaviour of scatter data in circular-domain. The detail description about application of circular features on laboratory slewing bearing data based PAA result is explained in $§ 5.3$.

Fig. 14 Illustration of PAA method as frequency alteration identification and time domain to circular domain transformation using the vibration data acquired on May $25^{\text {th }}$ (day 90).

\subsection{Circular Features Calculation}

To apply circular features calculation, the second at which class ' 1 ' orientation occurs is recorded. The time occurrence is transformed into angular dimension $\alpha$ using Eq. (12).

\section{Circular mean and mean resultant vector:}

The mean of vector $\alpha$ cannot be estimated using simple linear averaging data points. Since $\alpha$ is in angular directions, $\alpha$ are initially transformed to unit vectors in the two-dimensional plane by

$$
\begin{gathered}
Z_{i}=r \cos \alpha_{i} \\
\quad \text { or } \\
Z_{i}=r \sin \alpha_{i}
\end{gathered}
$$

where $r$ is the radius of two-dimensional circular plane and $r=1$ is used in this paper.

This is illustrated in Fig. 15(b), where all data points, $\alpha$ (occurrences) marked by blue circles lie on the unit sphere. As indicated further in Fig. 15(b), the x-coordinate of a point $\left(\alpha_{i}\right)$ corresponds to cosine of the angle and the y-coordinate to the sine. For ease of implementation in MATLAB, this transformation is written in the following equation

$$
Z_{i}=r\left(\cos \alpha_{i}+i \sin \alpha_{i}\right)=r \exp \left(i \alpha_{i}\right)
$$

After this transformation, the mean of $\bar{Z}$ can be computed from the vectors $Z_{i}$ by 


$$
\bar{Z}=\frac{1}{C} \sum_{i} Z_{i}
$$

where $C$ is the number of data points (occurrence). The vector $\bar{Z}$ is called mean resultant vector. To yield the circular mean $\bar{\alpha}$ use the built-in function angle in MATLAB to transform $\bar{Z}$ into the circular mean $\bar{\alpha}$. Moreover, $i$ indicates point in the circular domain where the correlation plot has right orientation (45 degree) or class ' 1 '.

\section{Resultant vector length:}

The length of the mean resultant vector is a fundamental quantity for the measurement of circular spread in circular domain [13]. The more concentrated the data sample is around the mean direction. The resultant vector length is estimated by

$$
R=\|\bar{Z}\|
$$

Circular variance:

The circular variance is closely related to the length of the mean resultant vector. It is defined as

$$
V=1-R
$$

Moreover [13] mentioned the different between the variance on a linear scale and the circular variance. The circular variance is bounded within the interval $[0,1]$. It indicates the spread of a data set. If all samples point lies on the angular scale with the same direction, the resultant vector will have length close to 1 and the circular variance will correspondingly be small. If the samples spread out evenly around the circle, the resultant vector will have length close to ' 0 ' and the circular variance will be close to maximal.

\section{Circular skewness:}

As the third order statistical moments, circular skewness measures the symmetry of distribution data with respect to the circular mean. It can be calculated as follows [11]

$$
m=\frac{1}{C} \sum_{i=1}^{c} \sin 2\left(\alpha_{i}-\bar{\alpha}\right)
$$




\section{Circular kurtosis:}

Similar to time domain kurtosis, circular kurtosis measure the degree of spread of the distribution around the peak. Kurtosis indicates the condition of bearing and provides potential damage detections at an earlier stage. When defects impact rolling element parts, it produces responsive signal that has probability density sharper than a normal condition [43]. Circular kurtosis can be estimated as [11]

$$
k=\frac{1}{C} \sum_{i=1}^{C} \cos 2\left(\alpha_{i}-\bar{\alpha}\right)
$$

A large positive sample value of $k$ close to one indicates a sharp distribution. Circular kurtosis value will increase when the class ' 1 ' ellipsoid orientation occurs more frequently and data is distributed uniformly in circular domain. On the contrary, kurtosis value will decrease when the data is few and distributed randomly. This makes the onset of bearing fault can be identified using circular kurtosis coupled with PAA data reduction method in slewing bearing case.

\subsection{Application of Circular Features on Laboratory Slewing Bearing Data Based PAA Result}

To get clearer detection of bearing condition, the ellipsoid classification is considered as bearing signal feature and presented in circular domain. In order to determine whether the shift is isolated instances or not, the ellipsoid orientation classification is conducted every second ( $\approx 6 \mathrm{deg}$ ) for one minute equivalent to reversible full rotation for $1 \mathrm{rpm}$. The highlights of circular domain transformation and circular features calculation result are depicted in Figs. 15 and 16. Figs. 15 and 16 show the difference of circular features result based on PAA method obtained on day $9^{\text {th }}$ and day $90^{\text {th }}$ of the bearing running time. The time domain signal representation in circular domain is shown in part (a). The vibration signal plotted in circular domain is the slewing bearing data in 30 seconds ( $\approx 180$ degree). After the application of PAA process with window size of 8 and the ellipse leastsquare fitting method, the ellipsoid shape classification based on the neighborhood correlation plot of PAA result is obtained. The classification result of every second is called 'occurrence'. The result of right ellipsoid occurrence ' 1 ' is shown in part (b) (shown in circle blue line). Finally, the four circular features based on the result plotted in part (b) are calculated to see the statistical behavior of the occurrences of the minute. The circular features calculation is presented in part (d). The process is then applied on lab slewing bearing data from February to August (138 days). The solid red line 
in Figs. 15 and 16(b) and (c) is the resultant vector length, $R$. The value of $R$ decreases when the data are spread out to angular domain.

Fig. 15 Lab slewing bearing data acquired on March 1 (day $9^{\text {th }}$ ): (a) Vibration data in circular domain; (b) Classification result shown the occurrence of right ellipsoid orientation (in radian); (c) Circular histogram (in degree); (d) Circular features calculation results.

Fig. 16 Lab slewing bearing data acquired on May 25 (day 90 ${ }^{\text {th }}$ ): (a) Vibration data in circular domain; (b) Classification result shown the occurrence of right ellipsoid orientation (in radian); (c) Circular histogram (in degree); (d) Circular features calculation results.

Fig. 17 Circular-domain features extraction results and comparable time-domain features from February to August 2007 (138 days).

The entire circular domain features calculation results from February to August (138 days period) are shown in Fig. 17. The circular domain features are compared to time domain features, features calculated from detail coefficient (D3) of wavelet decomposition and features calculated from the summation of the low IMF frequencies (from IMF 9 to the lowest IMF) of the EMD results. The further discussion is presented in §6. It is shown that the onset of bearing fault can be detected in angular domain allowing the engineer to make better scheduling of maintenance work. In detail, the circular features shows steady condition in the early stage of the bearing operation indicating the bearing is still in normal condition as shown in Figs. 17(a)-(d). When fault occurred, features show the peak value in day 90 indicates the onset of bearing fault and fluctuated starting from day 90 until 138 (end of August 2007). The onset of bearing fault is shown in four circular domain features presenting the consistency of circular domain features.

Since the proper window size is important in the detection of the frequency alteration, in this study we investigate the effect of different window size. In order to obtain the same data points in one frame, the window size should be the member of the factorials of sampling rate. In this study, the sampling rate is 4880 and thus the window size should be the member of the factorials of 4880 . Five different window sizes which are the member of factorials of 4880 were selected. The selected window sizes are 61, 40, 16, 8 and 4. In addition, eight different frequencies are also selected from $50 \mathrm{~Hz}$ to $225 \mathrm{~Hz}$ with the interval of $25 \mathrm{~Hz}$. Calculate the shift factor, $\phi$ as presented in Eq. (B3) using the predetermined window sizes and frequencies. The shift factor is used to identify the ellipsoid 
changes orientation from right to left or vice versa based on the threshold of $\phi=2$ (see Appendix B). The result of the calculated shift factors for different window sizes and different frequencies is presented in Table 5. It can be seen from Table 5 for window size of 8 , the shifting factor is greater than 2 for frequency less than $150 \mathrm{~Hz}$. On the contrary, the shifting factor is less than 2 for frequency greater than $150 \mathrm{~Hz}$. The clearer illustration is shown in Fig. 18. This condition is proved by the FFT result of May 25 as shown in Fig. 19, where the frequency of approximately $145 \mathrm{~Hz}$ is clearly shown. This frequency is much higher than the bearing fault frequencies presented in Table 5. We assumed that this frequency triggers the ellipsoid shifting orientation detected by PAA result.

Table 5 The effect of different window size on different unknown frequencies.

Fig. 18 Shift factor value for different frequency alteration and different window size.

Fig. 19 FFT of slewing bearing data on May 25 data i.e. 90 days after the start [9].

\section{Discussion}

A new circular domain analysis for slewing bearing condition monitoring based on PAA and circular domain features calculation has been introduced and presented. The method employed the different technique of calculation with previous and existing angular domain analysis as discussed in section 2. In order to present the impartial performance comparison between the proposed method and other methods, the proposed method is compared to time domain features calculation directly from vibration signal and features calculated from two advance signal processing: wavelet transform and EMD. The performance comparison is summarized in Table 6 and Table 7. The performance comparison is based on two key questions with regard to advanced and effective condition monitoring method for slewing bearing:

(1) Can the method identify the onset of bearing fault?

(2) Can the method present the progressive deterioration from the onset of bearing damage to the complete failure?

For question (1), it can be seen from Fig. 17 that the features of the proposed method show the maximum feature value in day 90 which is not observable from time domain features calculation and wavelet transform based features calculation. The maximum feature value in day 90 is also identified in EMD based features calculation results especially in variance and kurtosis features. We assumed that this phenomenon is the onset of slewing bearing fault. Therefore, for question (1), it is 
proved that the proposed method is able to identify the initial onset of bearing fault consistently as shown in four circular domain features compared to the other methods (see Fig. 17 and Table 6). However, the proposed method is unable to determine the failure degradation trend or progressive deterioration from the identified onset of bearing fault to complete failure. The progressive deterioration is more visible in features calculated from wavelet decomposition particularly in variance, skewness and kurtosis features; and time domain features especially in variance and kurtosis. Therefore, for question (2), the wavelet transform is superior to the other methods (see Fig. 17 and Table 7). During the slewing bearing lab experiment, the complete failure is unpredictable. Sudden burst vibration signal on September $2^{\text {nd }} 2007$ is occurred and the test-rig has to be shut down. To be able to discover the severe damage of slewing bearing and clarify the result of the features calculation, the slewing bearing was dismantled for inspection after the test-rig is shut down which is after day 138. Some of the defective regions can be clearly seen in Fig. 20.

Table 6 Comparison methods in identifying the initial onset of damage.

Table 7 Comparison methods in estimating the onset of degradation trend.

Fig. 20 (a) A view of damaged rollers in axial plane; (b) Outer raceway damage.

\section{Conclusion}

The merit and demerit of the proposed method has been presented with conscionable comparison to the three different methods. In this preliminary research, circular domain features have been shown to be potential condition monitoring parameters in reversible slow speed slewing bearing especially for the identification of the onset of bearing fault that serves as an early warning for preventive maintenance. This information will also be useful as bearing health prognostic tool. In addition, it worth noting that the combination of the proposed method and wavelet transform would be the appropriate method for slewing bearing condition monitoring and prognosis. Others potential circular features such as circular RMS and circular correlation will be investigated in future works.

\section{Acknowledgement}

The first author would like to thank to the University of Wollongong for the financial support through University Postgraduate Award (UPA) and International Postgraduate Tuition Award (IPTA) during this study. Also, Authors are grateful for the reviewers’ comments. 
Appendix A: The formula for calculating bearing fault frequencies [44]

- Fault frequency of outer ring:

$$
F_{O R}=\left|\frac{\left|I R_{r p m}-O R_{r p m}\right|}{2} \cdot\left[1-\frac{(\cos (\alpha)) \cdot d_{r}}{d_{m}}\right]\right| z
$$

- Fault frequency of inner ring:

$$
F_{I R}=\mid \frac{\left|R_{r p m}-O R_{r p m}\right|}{2} \cdot\left[1+\frac{(\cos (\alpha)) \cdot d_{r}}{d_{m}}\right] \cdot z
$$

- Fault frequency of rolling element:

$$
F_{R}=\left|\frac{\left|I R_{r p m}-O R_{r p m}\right|}{2} \cdot\left[\frac{d_{m}}{d_{r}}-\frac{(\cos (\alpha))^{2} \cdot d_{r}}{d_{m}}\right]\right|
$$

where $I R_{r p m}$ and $O R_{r p m}$ are the rotational speeds of the inner ring and outer ring. For $1 \mathrm{rpm}$ the value of $I R_{r p m}$ is 1 and the value of $O R_{r p m}$ is $0 . d_{m}$ denotes the mean bearing diameter, $d_{r}$ is diameter of the rolling element and $z$ is number of rolling elements.

Appendix B: The effect of window size to neighborhood correlation plot

The physical explanation why the right ellipsoid of neighborhood correlation plot will change and switch over is because the number of new data-reduced for a half sinusoidal signal is equal or less than 2. Through this appendix the answer is carried out empirically which also explain the phenomenon in Fig. 3, Fig. 4 and Fig. 5. Then the selection of window size of 8 in Lab slewing bearing data will be explained.

Figure $3(w=2)$ :

(a) In $1 \mathrm{~Hz}$ signal with sampling frequency of 64 for 1 second, the number of samples for one cycle sinusoidal signal is $64 / 1=64$ samples. The number of samples for a half sinusoidal signal is 64 samples/2 = 32 samples. If the window size is 2, thus in a half sinusoidal signal there are: 32 samples $/ 2$ window size $=16$ samples. (Note: right ellipsoid orientation)

(b) In $5 \mathrm{~Hz}$ signal with sampling frequency of 64 for 1 second, the number of samples for one cycle sinusoidal is $64 / 5=12.8$ samples. The number of samples for a half sinusoidal signal is 12.8 
samples $/ 2=6.4$ samples. If the window size is 2 , thus in a half sinusoidal signal there are: 6.4 samples/2 window size $=3.2$ samples. (Note: still right ellipsoid orientation)

Figure $4(w=4)$ :

(c) In $1 \mathrm{~Hz}$ signal with sampling frequency of 64 for 1 second, the number of samples for one cycle sinusoidal signal is $64 / 1=64$ samples. The number of samples for a half sinusoidal signal is 64 samples/2 = 32 samples. If the window size is 4 , thus in a half sinusoidal signal there are: 32 samples $/ 4$ window size $=8$ samples. (Note: still right ellipsoid orientation)

(d) In $5 \mathrm{~Hz}$ signal with sampling frequency of 64 for 1 second, the number of samples for one cycle sinusoidal is $64 / 5=12.8$ samples. The number of samples for a half sinusoidal signal is 12.8 samples $/ 2=6.4$ samples. If the window size is 4 , thus in a half sinusoidal signal there are: 6.4 samples/4 window size $=1.6$ samples. (Note: switch over to left ellipsoid orientation)

Figure $5(w=8)$ :

(e) In $1 \mathrm{~Hz}$ signal with sampling frequency of 64 for 1 second, the number of samples for one cycle sinusoidal signal is $64 / 1=64$ samples. The number of samples for a half sinusoidal signal is 64 samples/2 = 32 samples. If the window size is 8 , thus in a half sinusoidal signal there are: 32 samples/8 window size $=4$ samples. (Note: still right ellipsoid orientation)

(f) In $5 \mathrm{~Hz}$ signal with sampling frequency of 64 for 1 second, the number of samples for one cycle sinusoidal is $64 / 5=12.8$ samples. The number of samples for a half sinusoidal signal is 12.8 samples $/ 2=6.4$ samples. If the window size is 8 , thus in a half sinusoidal signal there are: 6.4 samples $/ 8$ window size $=0.8$ samples. (Note: change to left ellipsoid orientation)

\section{Appendix C: Shifting factor}

According to the empirical calculation above, the number of samples for a half sinusoidal signal, $S$ can be calculated by:

$$
S=0.5 \frac{f s}{\lambda}
$$


where, $f s$ is sampling frequency and $\lambda$ is frequency that trigger the change of ellipsoid orientation. Introducing an important dimensionless parameter called shifting factor, $\phi$ :

$$
\phi=\frac{S}{w}
$$

where $w$ is the window size. The different pattern of ellipsoid can be identified based on the value of, $\phi$ :

if $\phi>2$, the neighborhood correlation plot will right ellipsoid.

If $\phi \leq 2$, the neighborhood correlation plot will be left ellipsoid

Substitute Eq. (B1) to Eq. (B2) to calculate the shifting factor, $\phi$ :

$$
\phi=\frac{0.5 * \frac{f s}{\lambda}}{w}
$$

\section{References}

[1] D. Mba, R.H. Bannister, G.E. Findlay, Condition monitoring of low-speed rotating machinery using stress waves, Part 1, Proceedings of the Institution of Mechanical Engineers, Part E: Journal of Process Mechanical Engineering 213(3) (1999) 153-170.

[2] A.C.C. Tan, Y.-H. Kim, V. Kosse, Condition Monitoring of Low-Speed Bearing - A Review, Australian Journal of Mechanical Engineering, 6(1) (2008) 61-68.

[3] A. Widodo, E.Y. Kim, J.D. Son, B.S. Yang, A.C.C. Tan, D.S. Gu, B.K. Choi, J. Mathew, Fault Diagnosis of Low Speed Bearing based on Relevance Vector Machine and Support Vector Machine, Expert System with Application 36(3) (2009) 7252-7261.

[4] Y.H. Kim, A.C.C. Tan, J. Mathew, B.S. Yang, Condition monitoring of low speed bearings: A comparative study of the ultrasound technique versus vibration measurements, In proceedings of $1^{\text {st }}$ World Congress on Engineering Asset Management, Australia, 2006. 
[5] C.T. Yiakopoulos, K.C. Gryllias, I.A. Antoniadis, Rolling Element Bearing Fault Detection in Industrial Environments based on a K-means Clustering Approach, Expert System with Application 38 (2011) 2888-2911.

[6] W. Caesarendra, A. Widodo, P.H. Thom, B.S. Yang, J.D. Setiawan, Combined Probability Approach and Indirect Data-driven Method for Bearing Degradation Prognostics, IEEE Transactions on Reliability 60(1) (2011) 14-20.

[7] Y. Lei, Z. He, Y. Zi, A Combination of WKNN to Fault Diagnosis of Rolling Element Bearings, ASME Journal of Vibration and Acoustics 31 (2009) 064502-1 - 064502-6.

[8] A. Widodo, B.S. Yang, Machine health prognostics using survival probability and support vector machine, Expert Systems with Applications 38 (2011) 8430-8437.

[9] C.A.S. Moodie, An Investigation into the Condition Monitoring of Large Slow Speed Slew Bearings, Ph.D. thesis, University of Wollongong, Wollongong, 2009.

[10] N. Jamaludin, D. Mba, R.H. Banister, Condition monitoring of slow-speed rolling element bearings using stress waves, Proceedings of the Institution of Mechanical Engineers, Part E: Journal of Process Mechanical Engineering, (2001) 215-245.

[11] A. Pewsey, The Large-Sample Joint Distribution of Key Circular Statistics, Metrika 60(1) (2004) 25-32.

[12] N.I. Fisher, Statistical Analysis of Circular Data, Revised edition, Cambridge University Press, 1995.

[13] P. Berens, CircStat: A MATLAB Toolbox for Circular Statistics, Journal of Statistics Software 31(10) (2009) 1-21.

[14] M.F. Luo, J. Mathew, Angle domain analysis technique for monitoring machinery with varying speed, Centre for Machine Condition Monitoring Research Bulletin, Monash University, 5(1) (1993) 4.1-4.10.

[15] K.R. Fyfe, E.D.S. Munck, Analysis of computed order tracking, Mechanical Systems and Signal Processing 11 (2) (1997) 187-205.

[16] K.M. Bossley, R.J. McKendrick, C.J. Harris, C. Mercer, Hybrid computed order tracking, Mechanical Systems and Signal Processing 13 (4) (1999) 627-641.

[17] F. Bonnardot, R.B. Randall, J. Antoni, Enhanced unsupervised noise cancellation using angular resampling for planetary bearing fault diagnosis, International Journal of Acoustics and Vibration 9 (2) (2004) 51-60.

[18] L. Renaudin, F. Bonnardot, O. Musy, J.B. Doray, D. Réymond, Natural roller bearing fault detection by angular measurement of true instantaneous angular speed, Mechanical Systems and Signal Processing 24 (2010) 1998-2011. 
[19] F. Bonnardot, M. El Badaoui, R.B. Randall, J. Danière, F. Guillet, Use of the acceleration signal of a gearbox in order to perform angular resampling (with limited speed fluctuation), Mechanical Systems and Signal Processing 19 (2005) 766-785.

[20] F. Combet, L. Gelman, An automated methodology for performing time synchronous averaging of a gearbox signal without speed sensor, Mechanical Systems and Signal Processing 21 (2007) 2590-2606.

[21] F. Combet, R. Zimrov, A new method for the estimation of the instantaneous speed relative fluctuation in a vibration signal based on the short time scale transform, Mechanical Systems and Signal Processing 23 (2009) 1382-1397.

[22] L.F. Villa, A. Renones, J.R. Perán, L.J. de Miguel, Angular resampling for vibration analysis in wind turbines under non-linear speed fluctuation, Mechanical Systems and Signal Processing 25 (2011) 2157-2168.

[23] L.F. Villa, A. Renones, J.R. Perán, L.J. de Miguel, Statistical fault diagnosis based on vibration analysis for gear test-bench under non-stationary conditions of speed and load, Mechanical Systems and Signal Processing 29 (2012) 436-446.

[24] M. Akar, Detection of a static eccentricity fault in a closed loop driven induction motor by using the angular domain order tracking analysis method, Mechanical Systems and Signal Processing 34 (2013) 173-182.

[25] C. Kovacs, Condition monitoring of low speed systems, Maintenance Management Conference, Paper 32 (1990) 1-20.

[26] B.S. Yang, A. Widodo, Introduction of Intelligent Machine Fault Diagnosis and Prognosis, Nova Science Publishers Inc., New York.

[27] C.I. Daubechies, Ten Lectures on Wavelet, SIAM, Pennsylvania, USA.

[28] G. Niu, A. Widodo, J.D. Son, B.S. Yang, D.H. Hwang, D.S. Kang, Decision-level fusion based on wavelet decomposition for induction motor fault diagnosis using transient current signal, Expert Systems with Applications 35 (2008) 918-928.

[29] Z. Feng, M. Liang, F. Chu, Recent advances in time-frequency analysis methods for machinery fault diagnosis: A review with application examples, Mechanical Systems and Signal Processing 38 (2013) 165-205.

[30] N.E. Huang, Z. Shen, S.R. Long, M.C. Wu, H.H. Shih, Q. Zheng, N.C. Yen, C.C. Tung, H.H. Liu, The empirical mode decomposition and the Hilbert spectrum for nonlinear and nonstationary time series analysis, Proceedings of the Royal Society London A 454 (1998) 903995. 
[31] S. Braun, M. Feldman, Decomposition of non-stationary signals into varying time scales: Some aspects of the EMD and HVD methods, Mechanical Systems and Signal Processing 25 (2011) 2608-2630.

[32] Y. Lei, J. Lin, Z. He, M.J. Zuo, A review on empirical mode decomposition in fault diagnosis of rotating machinery, Mechanical Systems and Signal Processing 35 (2013) 108-126.

[33] G, Rilling, P. Flandrin, P. Gonçalves, On empirical mode decomposition and its algorithms, IEEE-EURASiP Workshop on Nonlinear Signal and image Processing, NSiP-03, Grado (1), June 2003.

[34] W. Caesarendra, P.B. Kosasih, A.K.Tieu, C.A.S. Moodie, B.K. Choi, Condition monitoring of naturally damaged slow speed slewing bearing based on ensemble empirical mode decomposition, Journal of Mechanical Science and Technology 27(8) (2013) 1-10.

[35] B.-K. Yi, C. Faloutsos, Fast Time Sequence Indexing for Arbitrary Lp Norms, Proceeding on $26^{\text {th }}$ VLBD Conference, Cairo, Egypt, 2000, 385-394.

[36] E. Keogh, K. Chakrabarti, M. Pazzani, Mehrotra, Dimensionality Reduction for Fast Similarity Search in Large Time Series Databases, Journal of Knowledge Information System (2000) 263-286.

[37] A. Fitzgibbon, M. Pilu, R.B. Fisher, Direct Least Square Fitting of Ellipses, IEEE Transactions on Pattern Analysis and Machine Intelligence 21(5) (1999) 476-480.

[38] P.L. Rosin, A Note on the Least Squares Fitting of Ellipses, Pattern Recognition Letters, no. 14, pp. 799-808, Oct. 1993.

[39] W. Gander, G.H. Golub, R. Strebel, Least-Square Fitting of Circles and Ellipses, BIT, no. 43, pp. 558-578, 1994.

[40] R. Haralick, R. Shapiro, Computer and Robot Vision, Vol. I (1992), Addison-Wesley, Reading, MA.

[41] F.L. Bookstein, Fitting conic sections to scattered data, Computer Graphics and Image Processing 9 (1979) 56-71.

[42] S. Hou, Y. Li, Z. Wang, M. Liang, A New Low-Frequency Resonance Sensor for Low Speed Roller Bearing Monitoring, ASME Journal of Vibrations and Acoustics 132 (2010) 014502-1 $-014502-8$.

[43] W. Caesarendra, A. Widodo, B.S. Yang, Application of Relevance Vector Machine and Logistic Regression for Machine Degradation Assessment, Mechanical Systems and Signal Processing 24 (2010) 1161-1171.

[44] P. Eschmann, L. Hasbargen, K. Weigand, Die Wälzlagerpraxis: Handbuch für die Berechnung und Gestaltung von Lagerungen, Publisher: R. Oldenburg, 1953. 
Table 1 Fault frequencies of slewing bearing (run at $1 \mathrm{rpm}$ and $4.5 \mathrm{rpm}$ for slewing bearing test-rig and slewing bearing Bridge Reclaimer, respectively).

\begin{tabular}{lcccc}
\hline \multirow{2}{*}{ Defect mode } & \multicolumn{3}{c}{ Fault frequencies (Hz) (calculation is given in Appendix A) } \\
\cline { 2 - 5 } & \multicolumn{2}{c}{ Slewing bearing test-rig } & \multicolumn{2}{c}{ Slewing bearing Bridge Reclaimer } \\
\cline { 2 - 5 } & Axial & Radial & Axial & Radial \\
\hline Outer ring (BPFO) & 1.32 & 0.55 & 13.41 & 11.38 \\
Inner ring (BPFI) & 1.37 & 0.55 & 13.58 & 11.56 \\
Rolling element (BSF) & 0.43 & 0.54 & 5.65 & 4.87 \\
\hline \hline
\end{tabular}

Table 2 EMD result of vibration data on March $1^{\text {st }} 2007$.

\begin{tabular}{lcc}
\hline \multirow{2}{*}{ EMD result } & \multicolumn{2}{c}{ Decomposition information } \\
\cline { 2 - 3 } & Frequency $(\mathrm{Hz})$ & RMS amplitude $(\mathrm{mV})$ \\
\hline Original vibration data & - & 3.150 \\
IMF 1 & 703.511 & 1.636 \\
IMF 2 & 688.399 & 0.814 \\
IMF 3 & 339.951 & 1.476 \\
IMF 4 & 135.495 & 1.813 \\
IMF 5 & 84.989 & 1.072 \\
IMF 6 & 41.812 & 0.542 \\
IMF 7 & 20.336 & 0.470 \\
IMF 8 & 8.183 & 0.415 \\
IMF 9 & 3.973 & 0.322 \\
IMF 10 & 2.058 & 0.228 \\
IMF 11 & 1.142 & 0.151 \\
IMF 12 & 0.544 & 0.094 \\
IMF 13 & 0.284 & 0.057 \\
IMF 14 & 0.100 & 0.002 \\
\hline
\end{tabular}


Table 3 EMD result of vibration data on May 25 2007.

\begin{tabular}{lcc}
\hline \multirow{2}{*}{ EMD result } & \multicolumn{2}{c}{ Decomposition information } \\
\cline { 2 - 3 } & Frequency $(\mathrm{Hz})$ & RMS \\
\hline Original vibration data & - & 16.940 \\
IMF 1 & 667.759 & 10.669 \\
IMF 2 & 550.533 & 9.966 \\
IMF 3 & 270.841 & 8.992 \\
IMF 4 & 132.749 & 3.686 \\
IMF 5 & 68.341 & 2.070 \\
IMF 6 & 34.238 & 1.589 \\
IMF 7 & 17.494 & 1.219 \\
IMF 8 & 8.620 & 0.940 \\
IMF 9 & 4.270 & 0.613 \\
IMF 10 & 28.112 & 0.678 \\
IMF 11 & 1.035 & 0.406 \\
IMF 12 & 0.459 & 0.574 \\
IMF 13 & 0.295 & 0.393 \\
IMF 14 & 0.099 & 0.458 \\
IMF 15 & 0.096 & 0.279 \\
\hline
\end{tabular}

Table 4 EMD result of vibration data on August 30 2007 .

\begin{tabular}{lcc}
\hline \multirow{2}{*}{ EMD result } & \multicolumn{2}{c}{ Decomposition information } \\
\cline { 2 - 3 } & Frequency (Hz) & RMS amplitude (mV) \\
\hline Original vibration data & - & 6.919 \\
IMF 1 & 651.717 & 1.822 \\
IMF 2 & 679.863 & 1.164 \\
IMF 3 & 245.924 & 5.467 \\
IMF 4 & 121.320 & 4.137 \\
IMF 5 & 63.555 & 1.289 \\
IMF 6 & 28.892 & 0.745 \\
IMF 7 & 13.682 & 0.559 \\
IMF 8 & 6.465 & 0.436 \\
IMF 9 & 3.054 & 0.331 \\
IMF 10 & 1.374 & 0.222 \\
IMF 11 & 0.682 & 0.131 \\
IMF 12 & 0.332 & 0.041 \\
IMF 13 & 0.099 & 0.023 \\
\hline
\end{tabular}


Table 5 The effect of different window size on different unknown frequencies.

\begin{tabular}{ccccccccc}
\hline \hline & \multicolumn{7}{c}{ Shift factor, $\phi$ for different unknown frequency alteration and different } \\
Window size, $w$ & \multicolumn{7}{c}{ window size } \\
\cline { 2 - 9 } & $50 \mathrm{~Hz}$ & $75 \mathrm{~Hz}$ & $100 \mathrm{~Hz}$ & $125 \mathrm{~Hz}$ & $150 \mathrm{~Hz}$ & $175 \mathrm{~Hz}$ & $200 \mathrm{~Hz}$ & $225 \mathrm{~Hz}$ \\
\hline$w=61$ & 0.8 & 0.533 & 0.4 & 0.32 & 0.267 & 0.229 & 0.2 & 0.178 \\
$w=40$ & 1.22 & 0.813 & 0.61 & 0.488 & 0.407 & 0.349 & 0.305 & 0.271 \\
$w=16$ & 3.05 & 2.033 & 1.525 & 1.22 & 1.017 & 0.871 & 0.763 & 0.678 \\
$w=8$ & 6.1 & 4.067 & 3.05 & 2.44 & $\mathbf{2 . 0 3 3}$ & 1.743 & 1.525 & 1.356 \\
$w=4$ & 12.2 & 8.133 & 6.1 & 4.88 & 4.067 & 3.486 & 3.05 & 2.71 \\
\hline \hline
\end{tabular}

Table 6 Comparison methods in identifying the initial onset of damage.

\begin{tabular}{lcccc}
\hline \multirow{2}{*}{ Feature } & \multicolumn{4}{c}{ Methods comparison } \\
\cline { 2 - 5 } Mean & Circular-domain & Time-domain & Wavelet decomposition & EMD \\
Variance & & & &
\end{tabular}

$\nabla$ Indicates clearly visible

X Indicates not visible

Table 7 Comparison methods in estimating the onset of degradation trend.

\begin{tabular}{|c|c|c|c|c|}
\hline \multirow{2}{*}{ Feature } & \multicolumn{4}{|c|}{ Methods comparison } \\
\hline & Circular-domain & Time-domain & Wavelet decomposition & EMD \\
\hline Mean & $X$ & 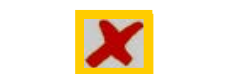 & $x$ & $Y$ \\
\hline Variance & Y & $V$ & E & \\
\hline Skewness & M & & V & N \\
\hline Kurtosis & $M$ & & T & $x$ \\
\hline
\end{tabular}

Indicates clearly visible

Indicates not visible 


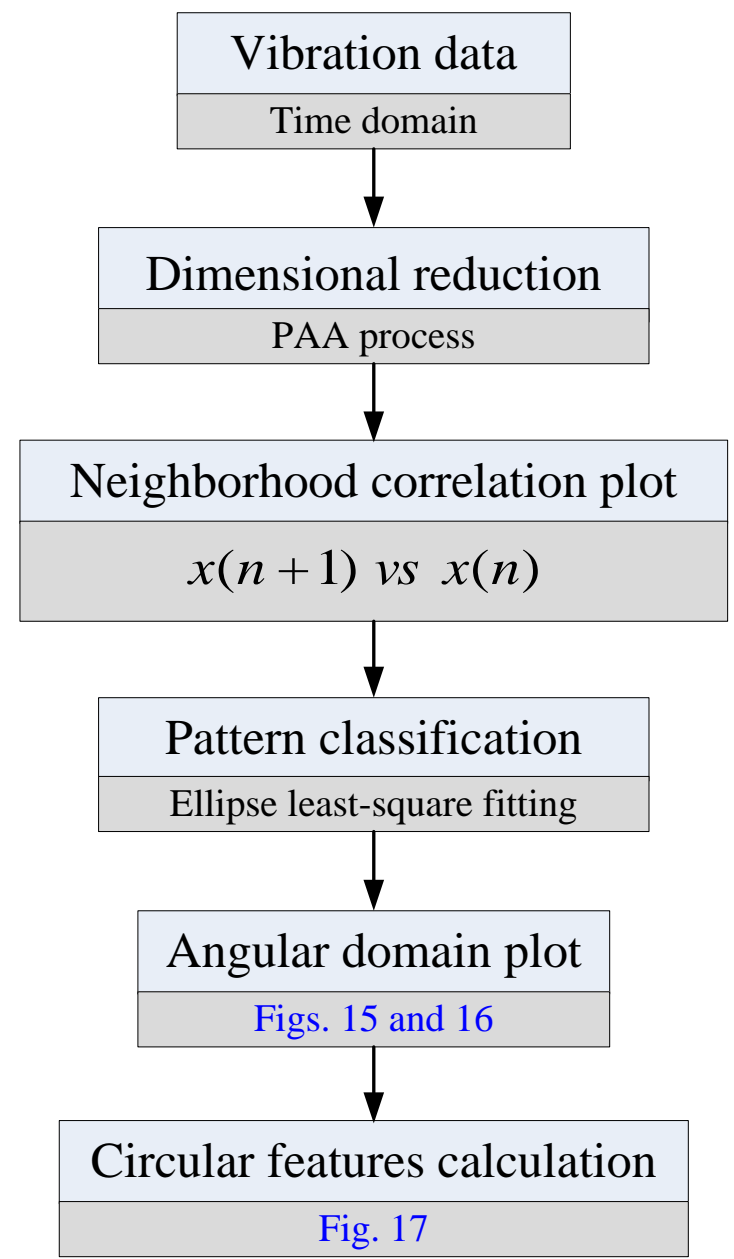

Fig. 1 Diagram of circular domain features extraction method. 


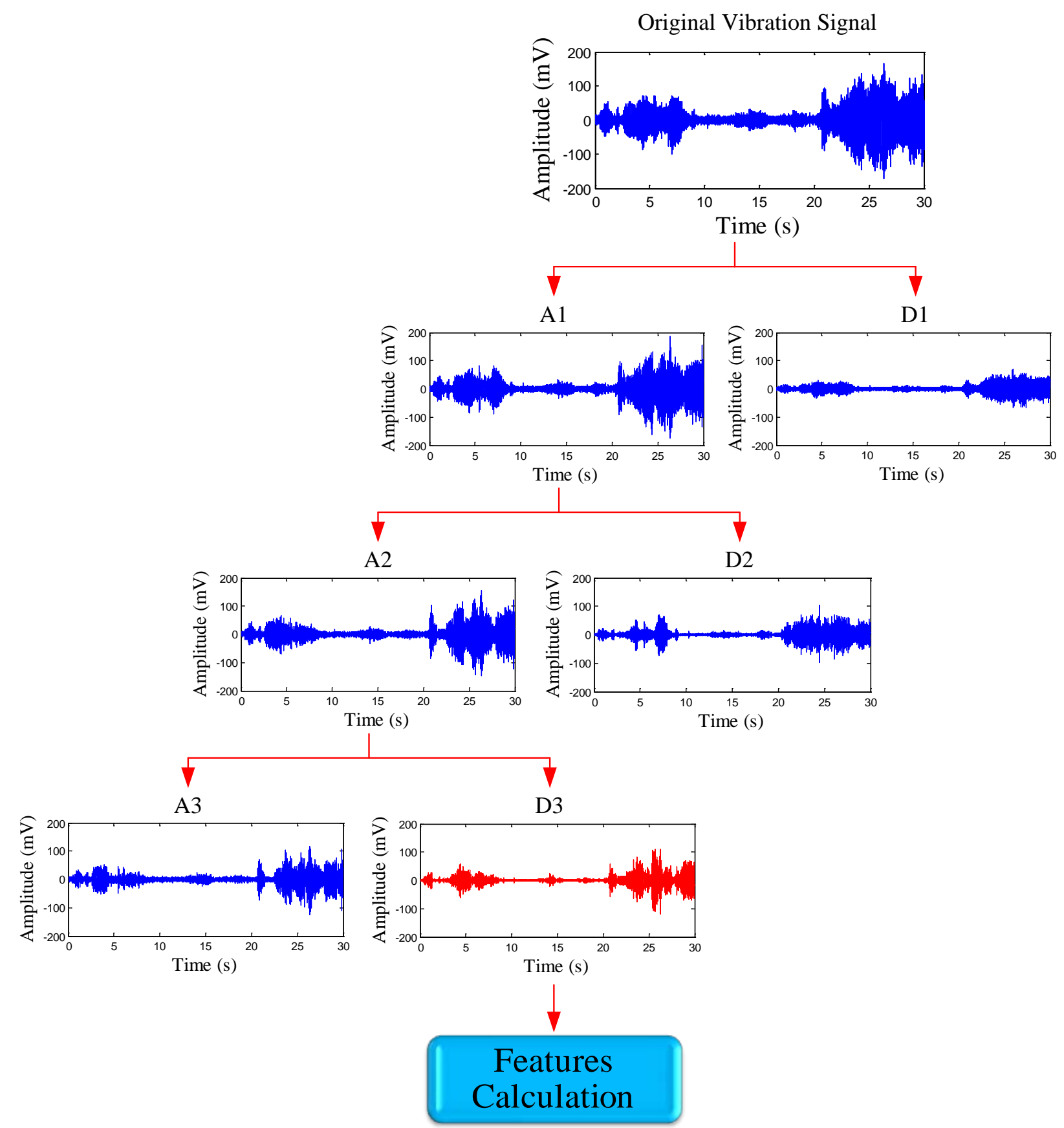

Fig. 2 Three level wavelet decompositions of the slewing bearing data (acquired on May $25^{\text {th }}$ ). 
Original vibration data
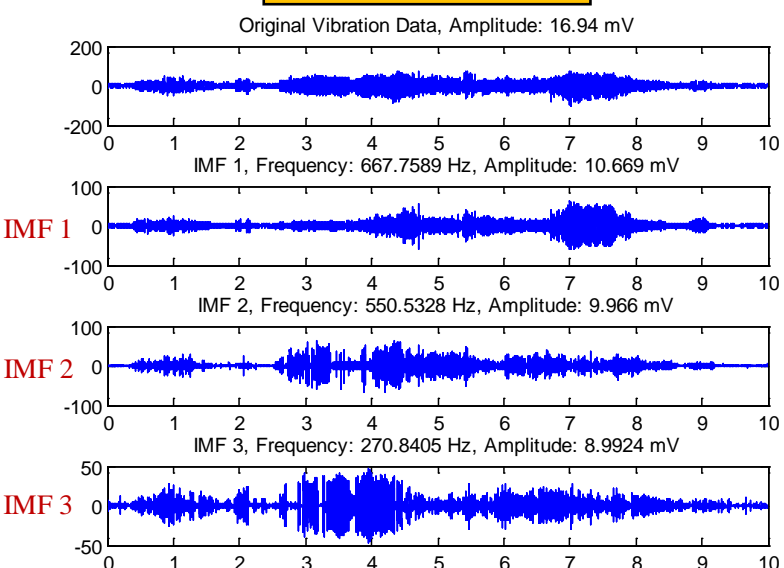

IMF 8, Frequency: $8.6203 \mathrm{~Hz}$, Amplitude: $0.94046 \mathrm{mV}$

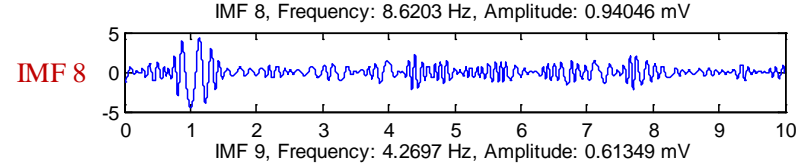

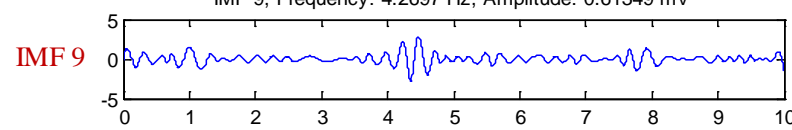
IMF 10, Frequency: $28.1118 \mathrm{~Hz}$, Amplitude: $0.67829 \mathrm{mV}$

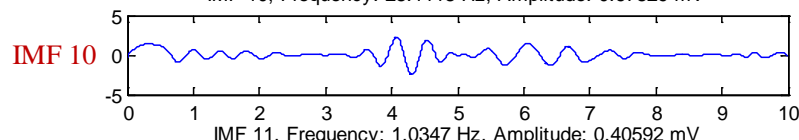

IMF 11

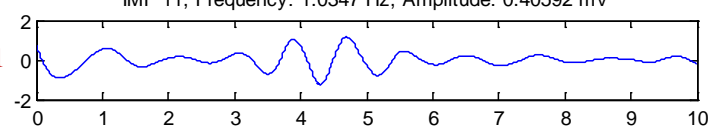

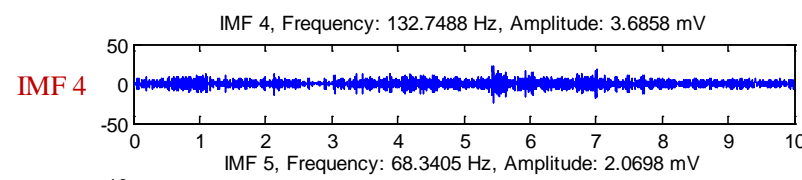

IMF 5

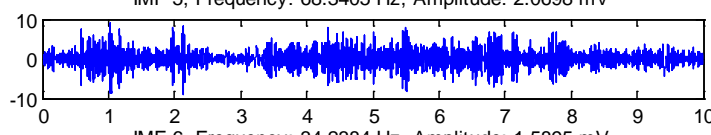
IMF 6, Frequency: $34.2384 \mathrm{~Hz}$, Amplitude: $1.5895 \mathrm{mV}$

IMF 6

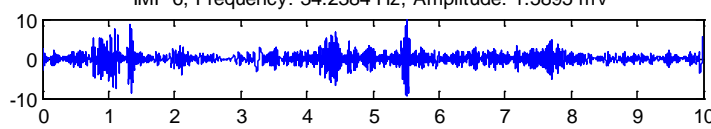

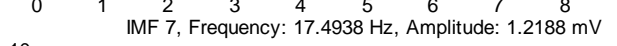

IMF 7
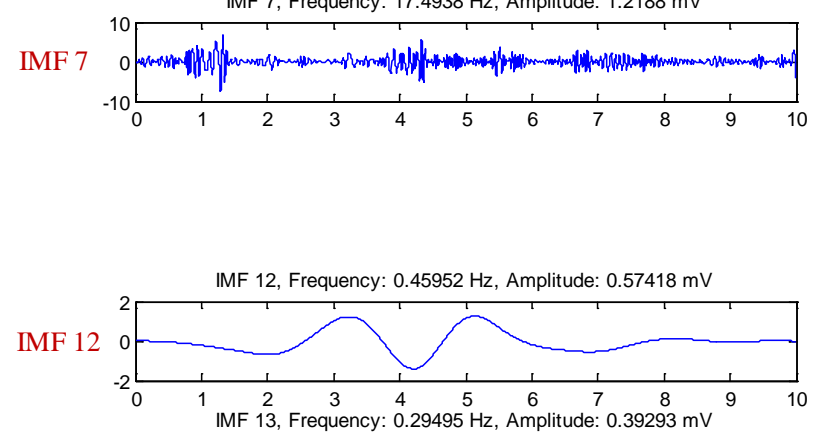

IMF 13

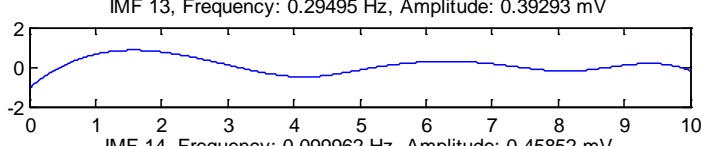

IMF 14

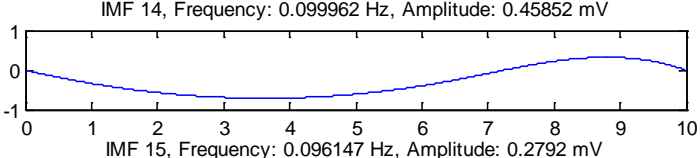

IMF 15

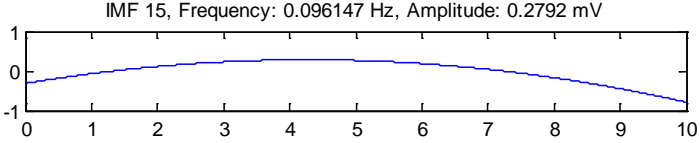

Fig. 3 EMD results of slewing bearing data (vibration data on May $25^{\text {th }}$ ). 


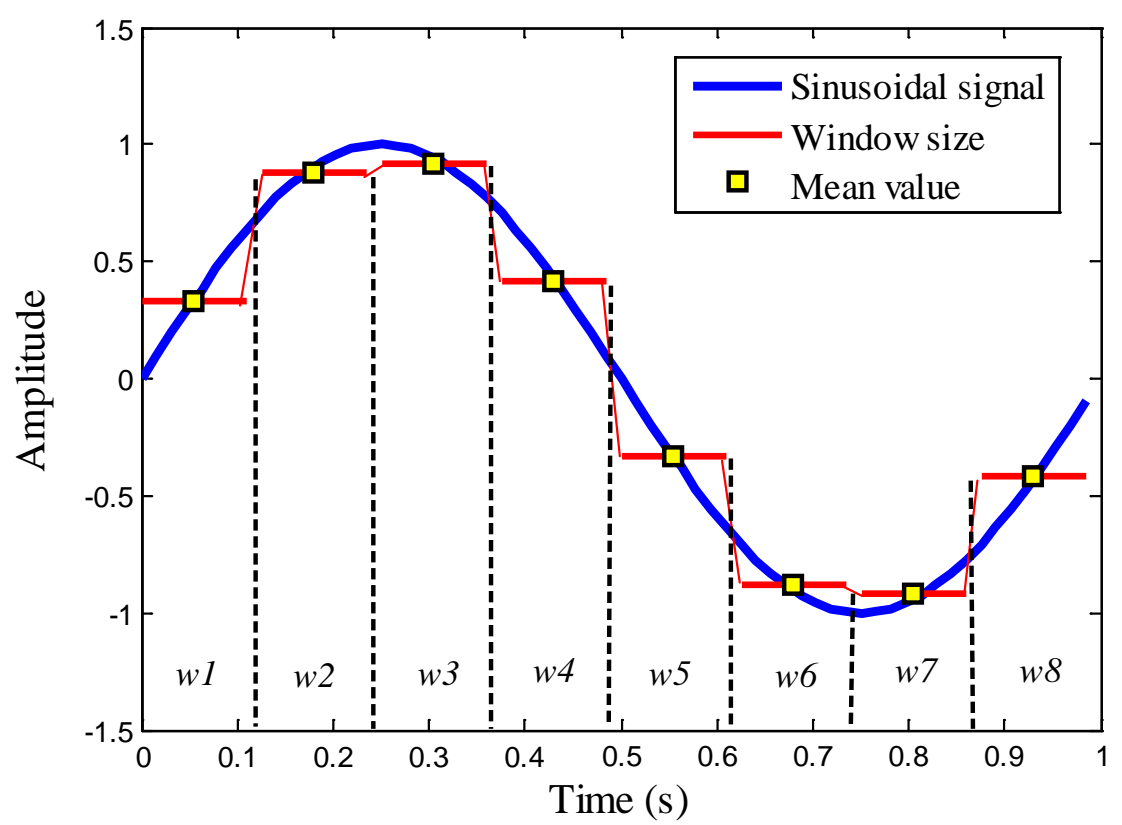

Fig. 4 One cycle of sinusoidal signal $(1 \mathrm{~Hz})$ sampled with $64 \mathrm{~Hz}$ sampling frequency is shown with solid blue curve. The data is divided into 8 frames (solid red line). The calculated mean value of sampled data in each frame is shown in yellow square shape. All mean values notated by the vector of $\mathbf{x}=\left(x_{1}, x_{2}, \ldots, x_{8}\right)$ is the PAA result. The index $w 1, w 2, \ldots w 8$ is the equal window size or "frame".
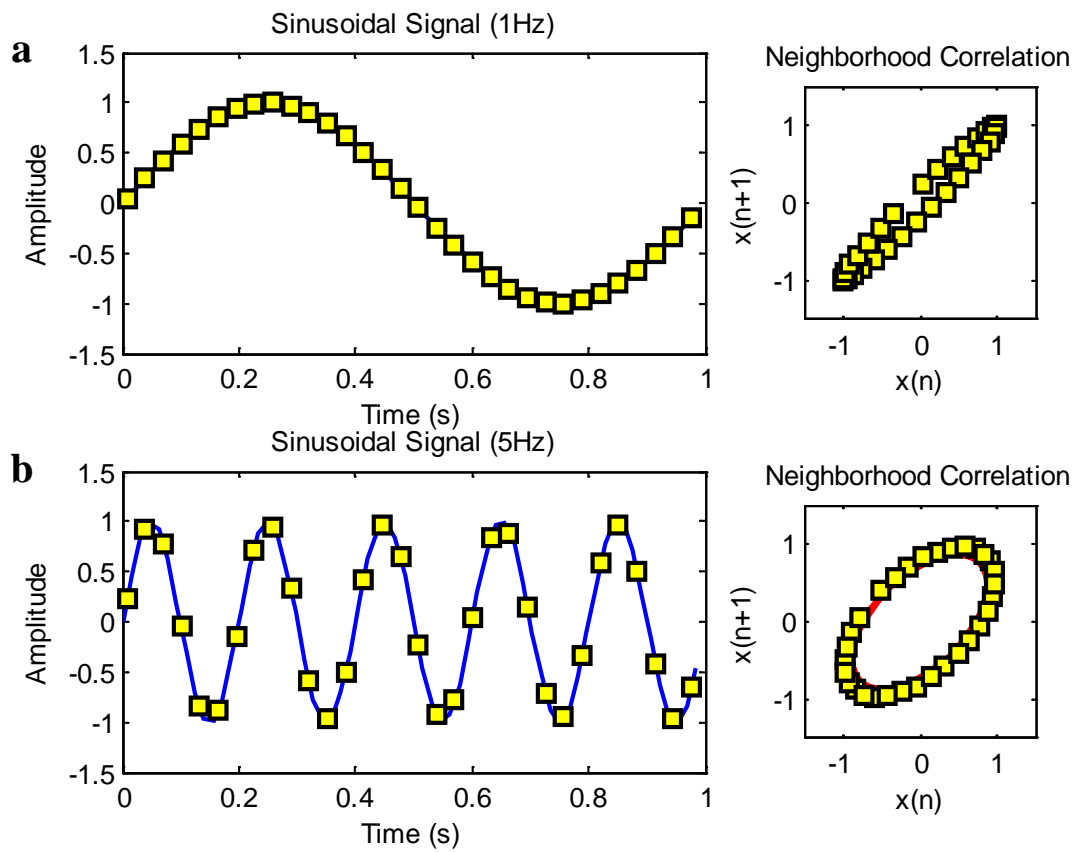

Fig. 5 Neighborhood correlation plots of $1 \mathrm{~Hz}$ and $5 \mathrm{~Hz}$ sinusoidal signals with window size $=2$. 

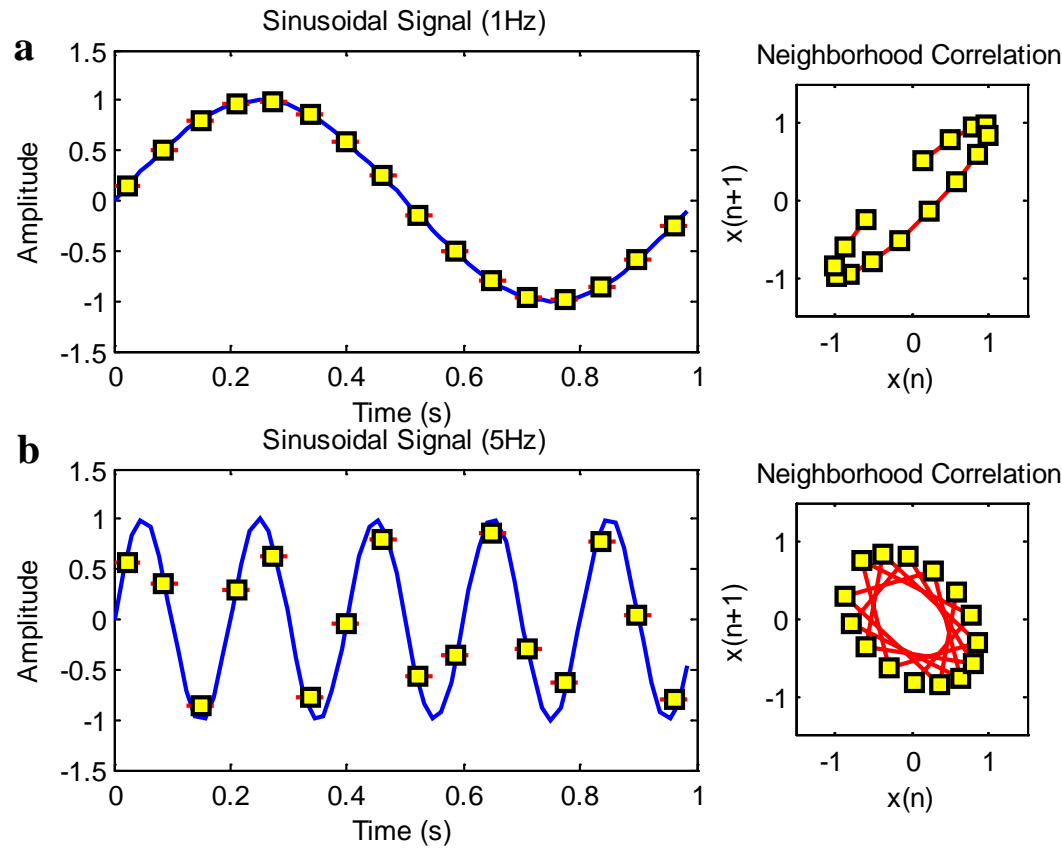

Fig. 6 Neighborhood correlation plots of $1 \mathrm{~Hz}$ and $5 \mathrm{~Hz}$ sinusoidal signals with window size $=4$.
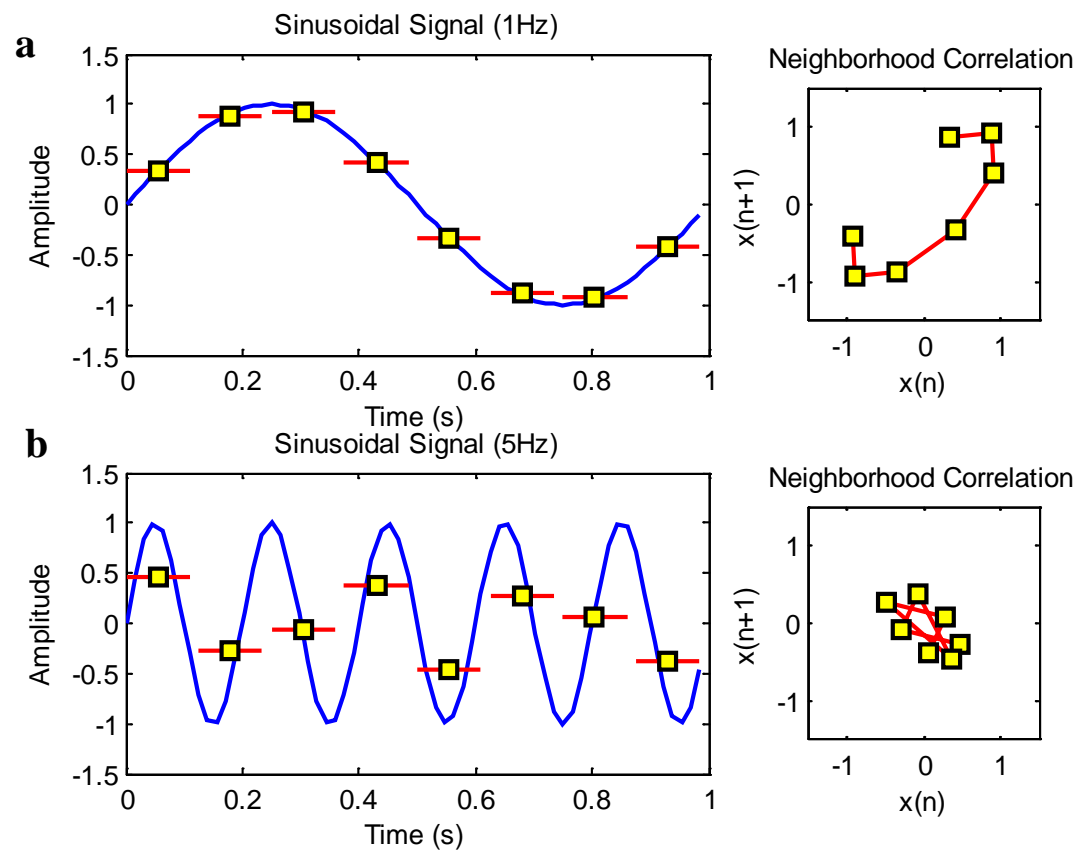

Fig. 7 Neighborhood correlation plots of $1 \mathrm{~Hz}$ and $5 \mathrm{~Hz}$ sinusoidal signals with window size $=8$. 
a

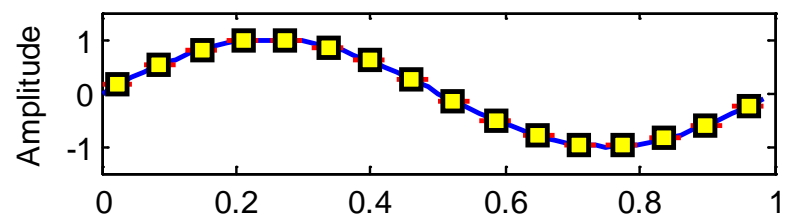

b

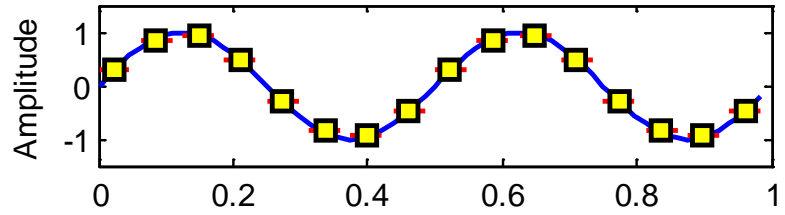

C

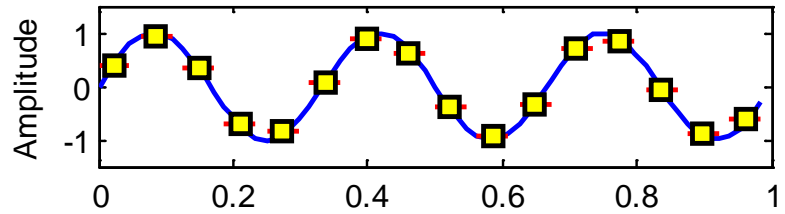

d

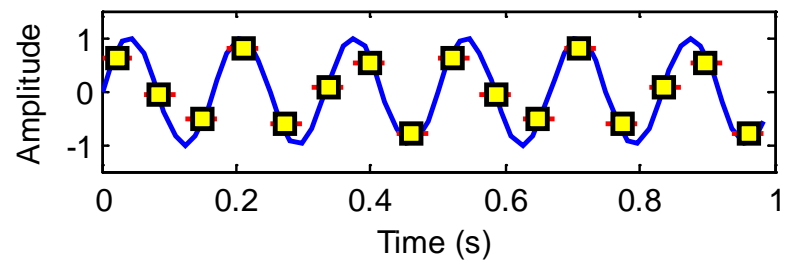

Neighborhood Correlation
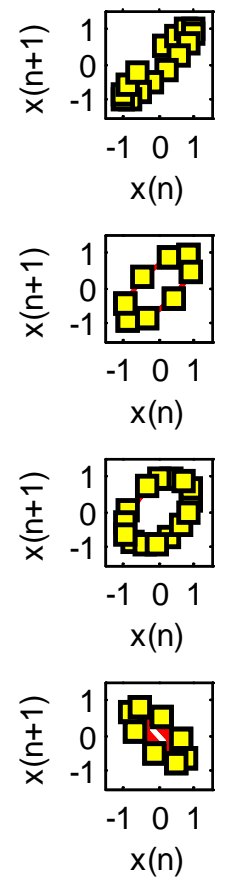

Fig. 8 Sinusoidal signal and PAA process (left) and neighborhood correlation plot based PAA result (right) for different frequencies with fix window size of 4: (a) $1 \mathrm{~Hz}$; (b) $2 \mathrm{~Hz}$; (c) $3 \mathrm{~Hz}$; (d) $6 \mathrm{~Hz}$. 


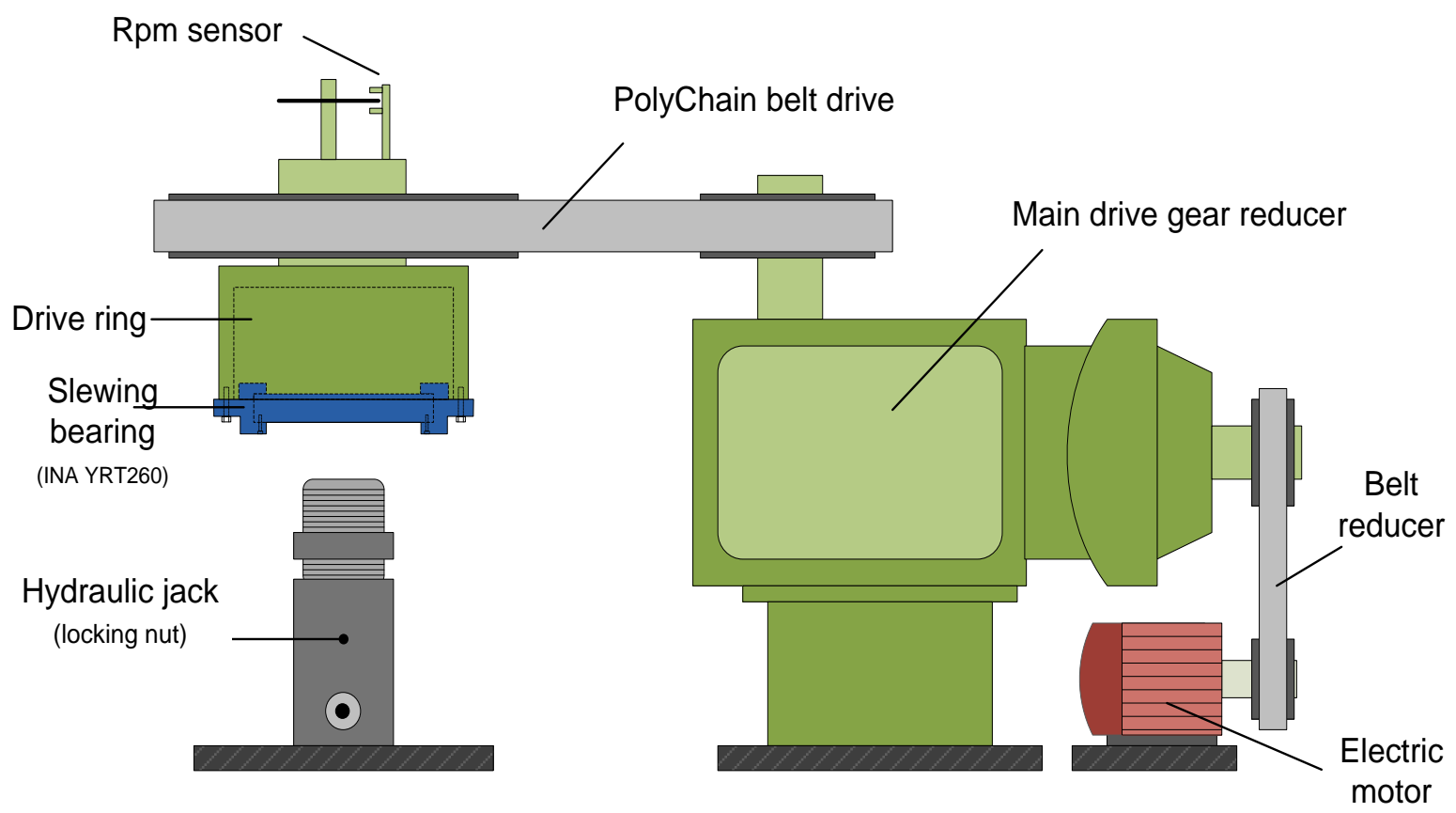

(a)

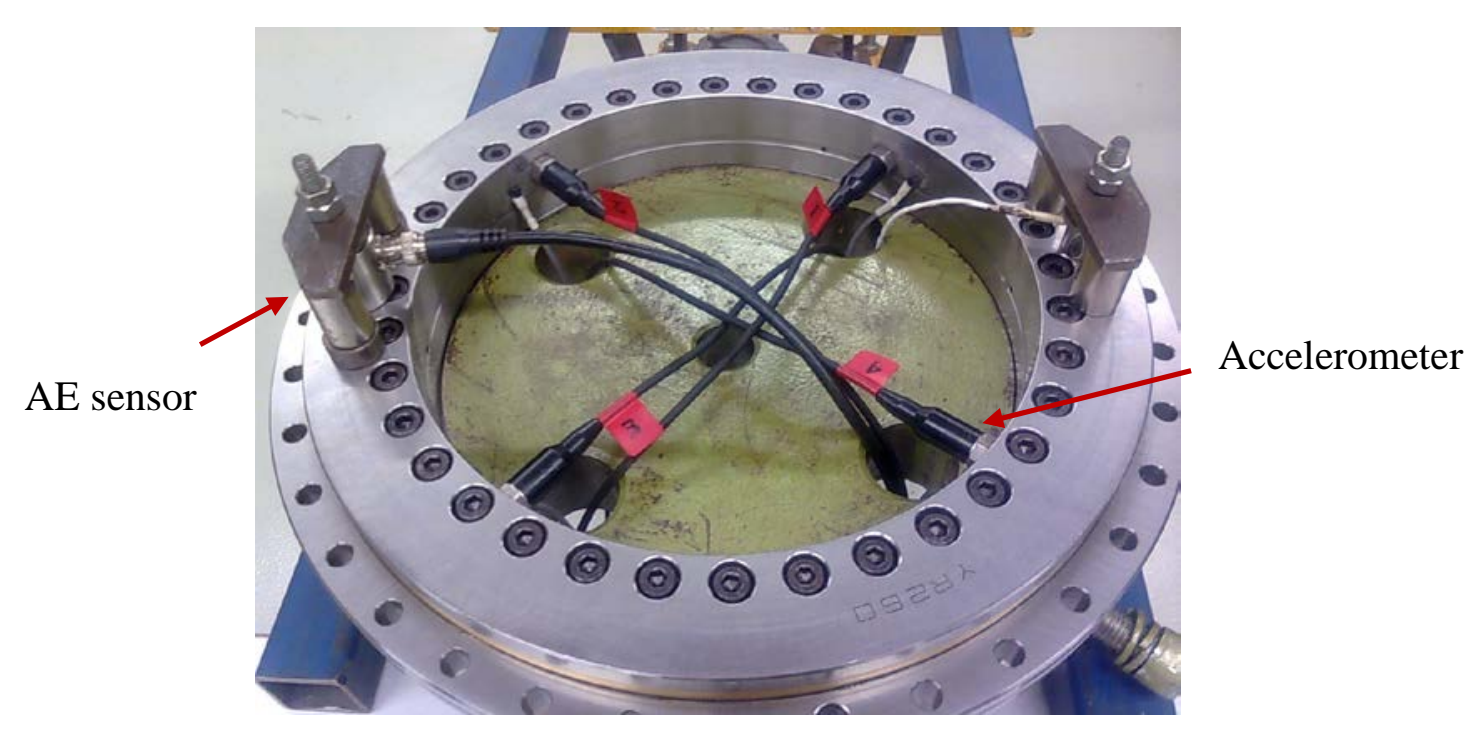

(b)

Fig. 9 (a) Schematic of lab slewing bearing rig; (b) Slewing bearing and sensors placement. 

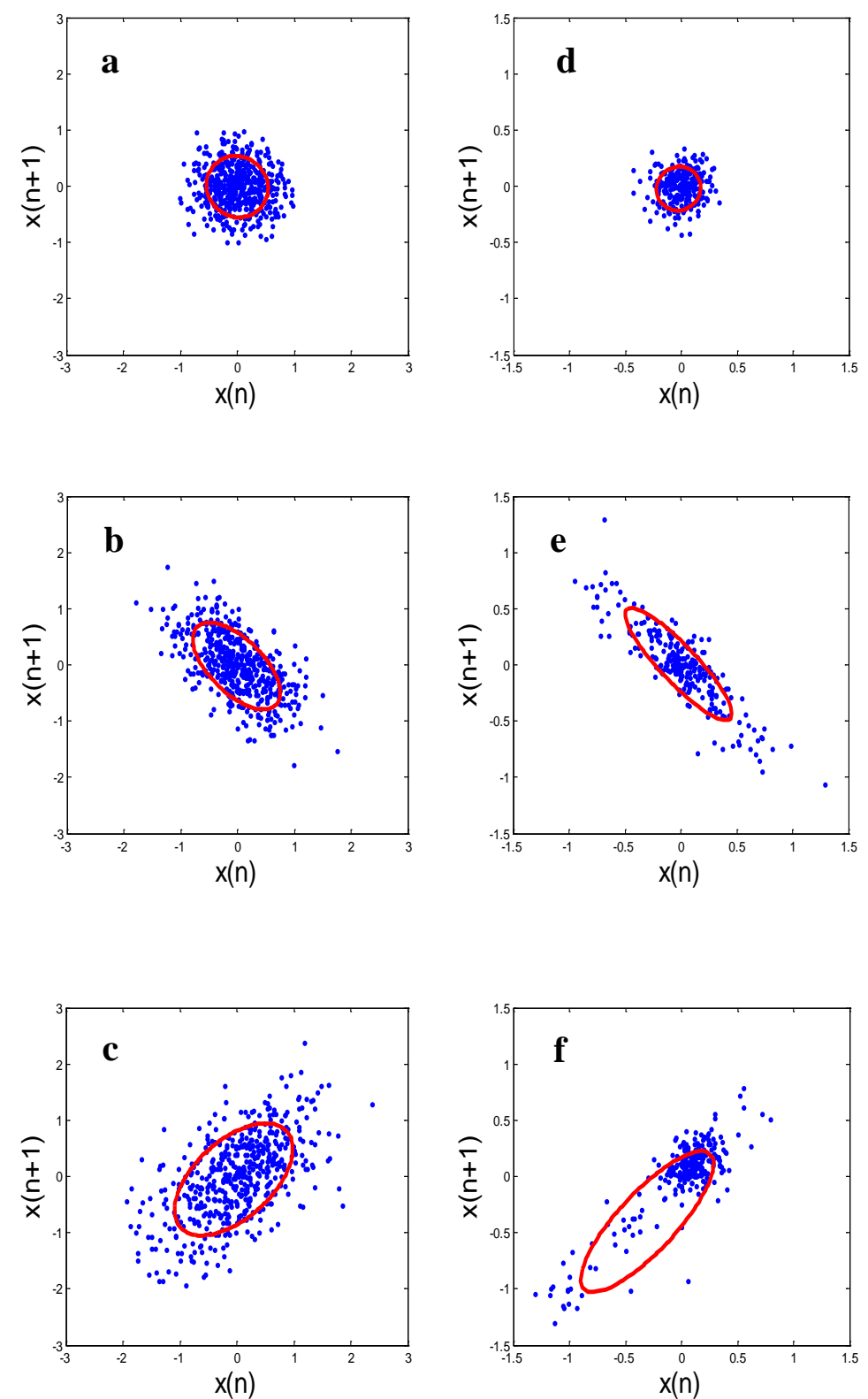

Fig. 10 Results of PAA data reduction of the lab test data: (a) March 2007; (b) May 2007; (c) August 2007; and results of Coal Bridge Reclaimer data: (d) May 2004; (e) January 2005; (f) September 2006. 
a

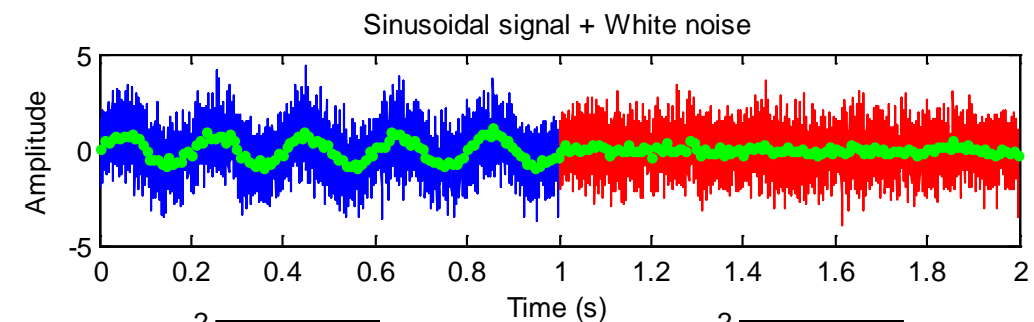

b
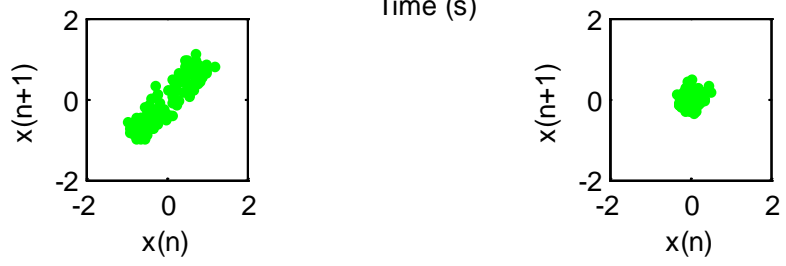

- Sampling frequency : 4880

- Sampling frequency : 4880

- Window size : 40

- Window size : 40

- PAA data reduced : 122

- PAA data reduced : 122

- Amplitude ratio : 0.56296

- Amplitude ratio : 0.07037

Fig. 11 (a) Simulated 5Hz signal plus white noise with different amplitude ratio; (b) PAA result or data reduced representation plotted in neighborhood correlation plot.

a

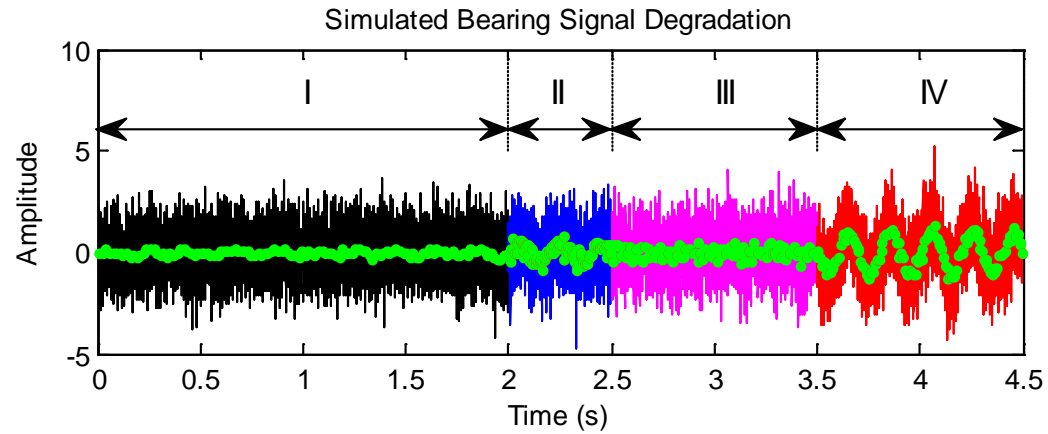

b
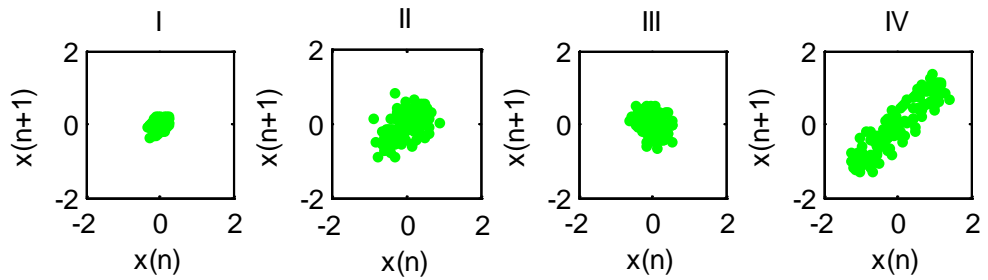

Fig. 12 Progression of simulated bearing signal degradation and the detection of fault occurrence using PAA. This signal is simulated with sampling frequency of 4880 and PAA window size of 40. Stage I: $5 \mathrm{~Hz}$ signal + white noise with amplitude ratio is 0.1 . Stage II: $5 \mathrm{~Hz}$ signal + white noise with amplitude ratio of 0.4 . Stage III: $55 \mathrm{~Hz}$ signal + white noise with amplitude ratio of 0.4 . Stage IV: $5 \mathrm{~Hz}$ signal + white noise with amplitude ratio of 1 . The four neighborhood plots show the evolution of the ellipsoid and its orientation change. 
Vibration slewing bearing signal
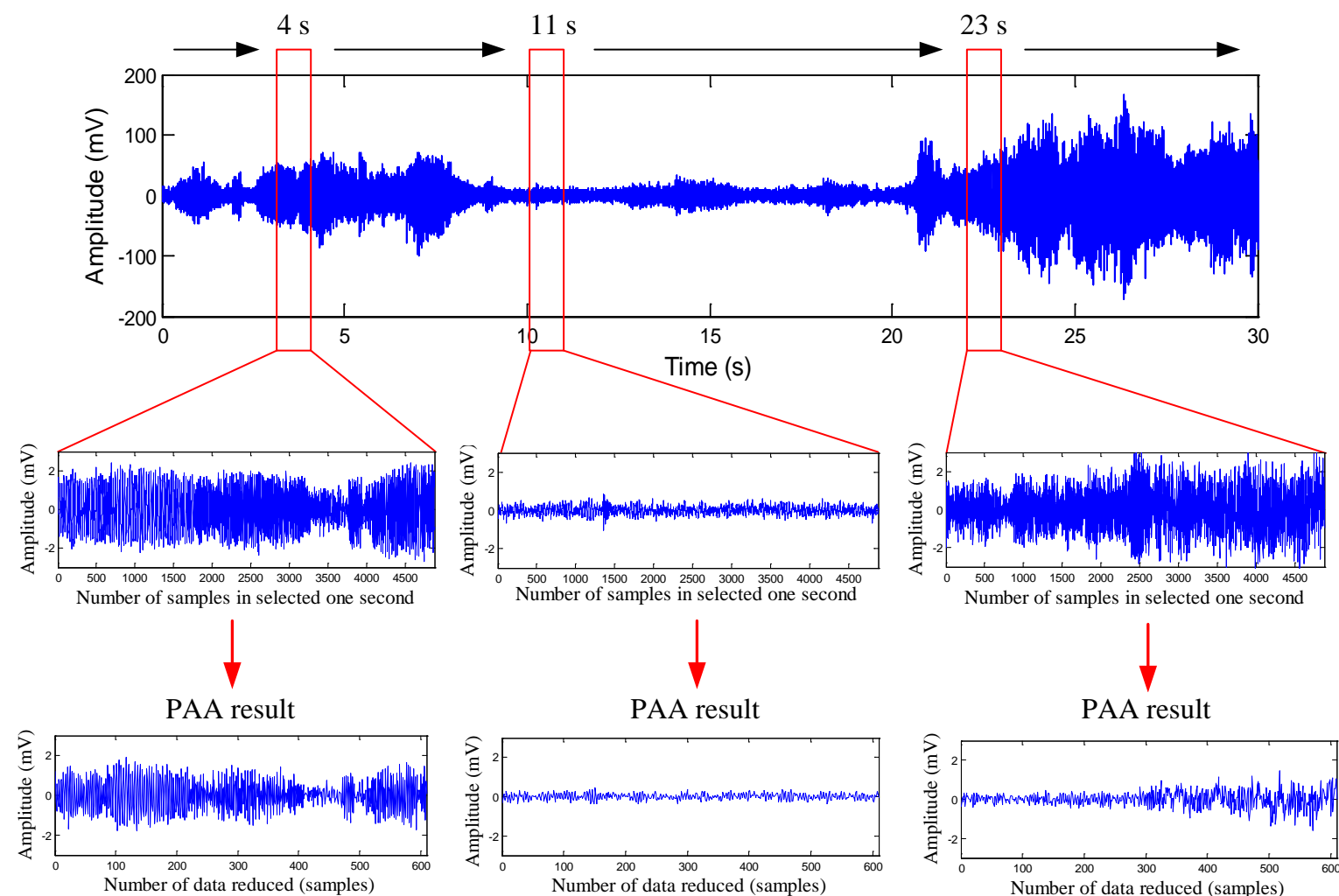

PAA result

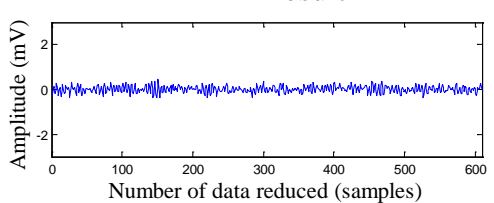

PAA result

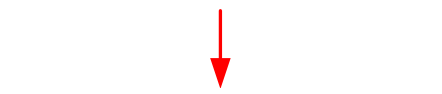

Neigborhood correlation plot

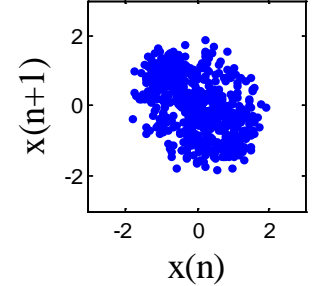

Neigborhood correlation plot
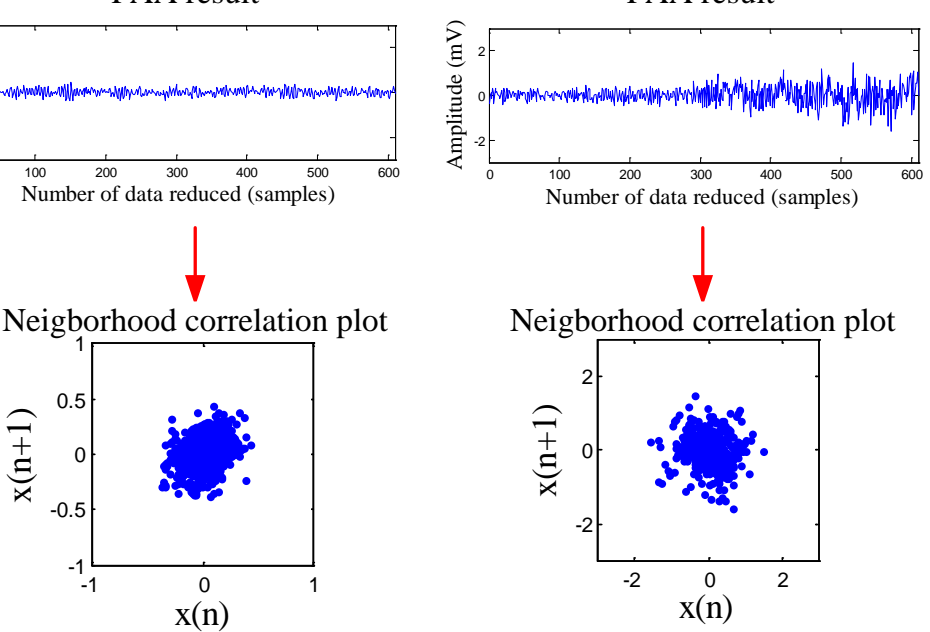

Number of data reduced (samples)

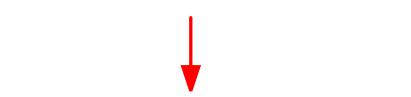

Neigborhood correlation plot

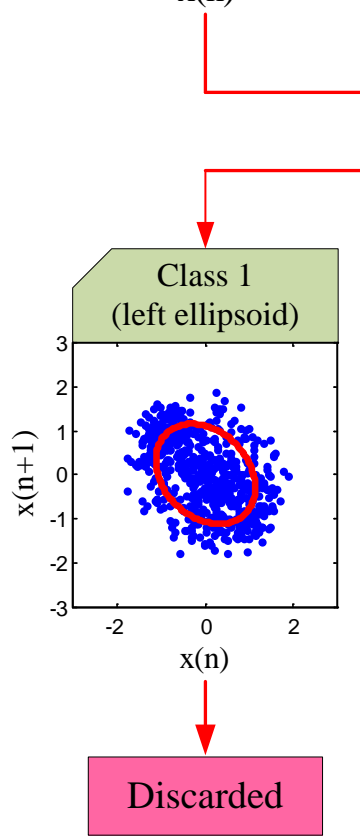

Pattern classification

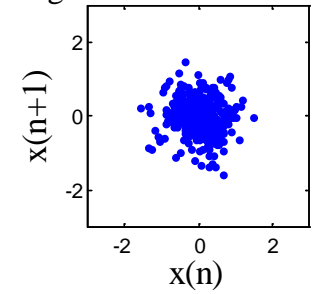

(n)

\section{Pattem classification}
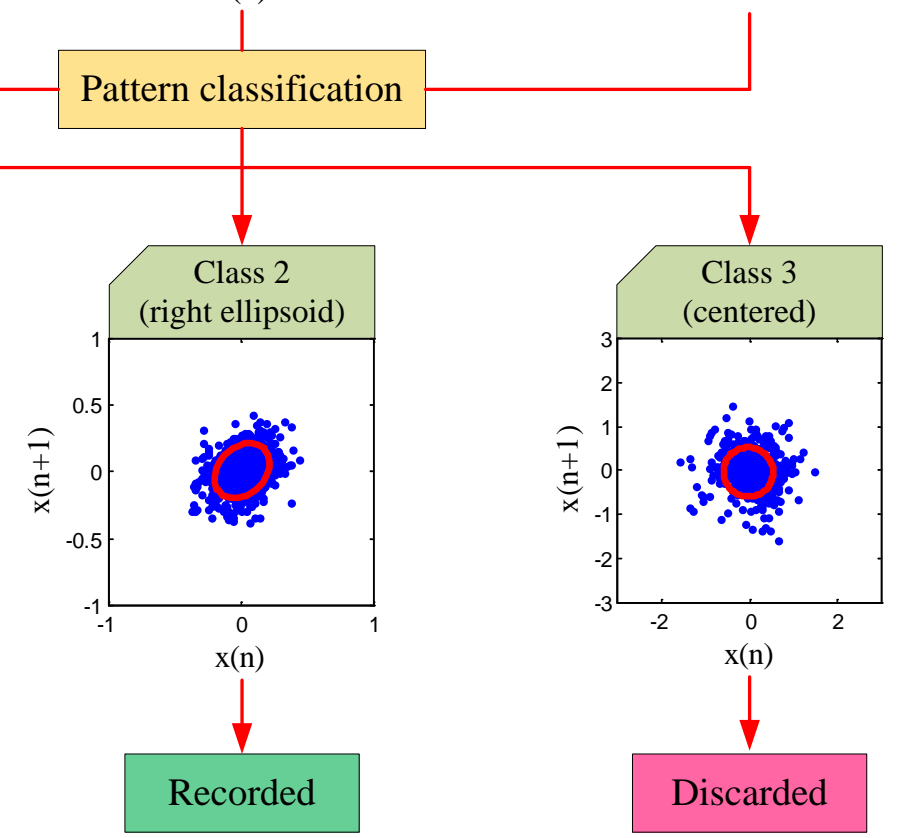

Fig. 13 Illustration of signal processing based on PAA method and ellipse least-square fitting method using vibration data acquired on May $25^{\text {th }}$ (day 90). 

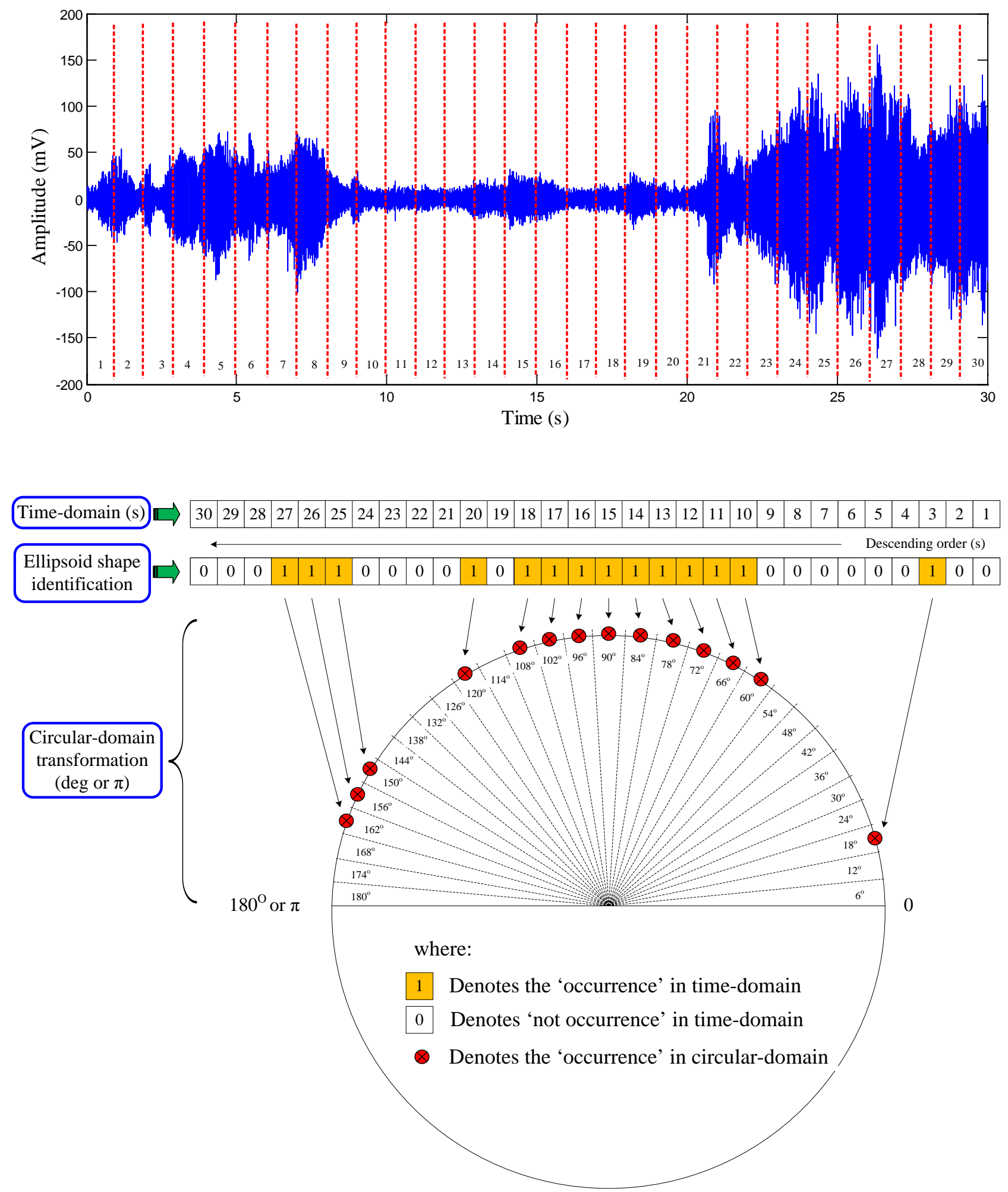

Fig. 14 Illustration of PAA method as frequency alteration identification and time domain to circular domain transformation using the vibration data acquired on May 25 ${ }^{\text {th }}$ (day 90). 

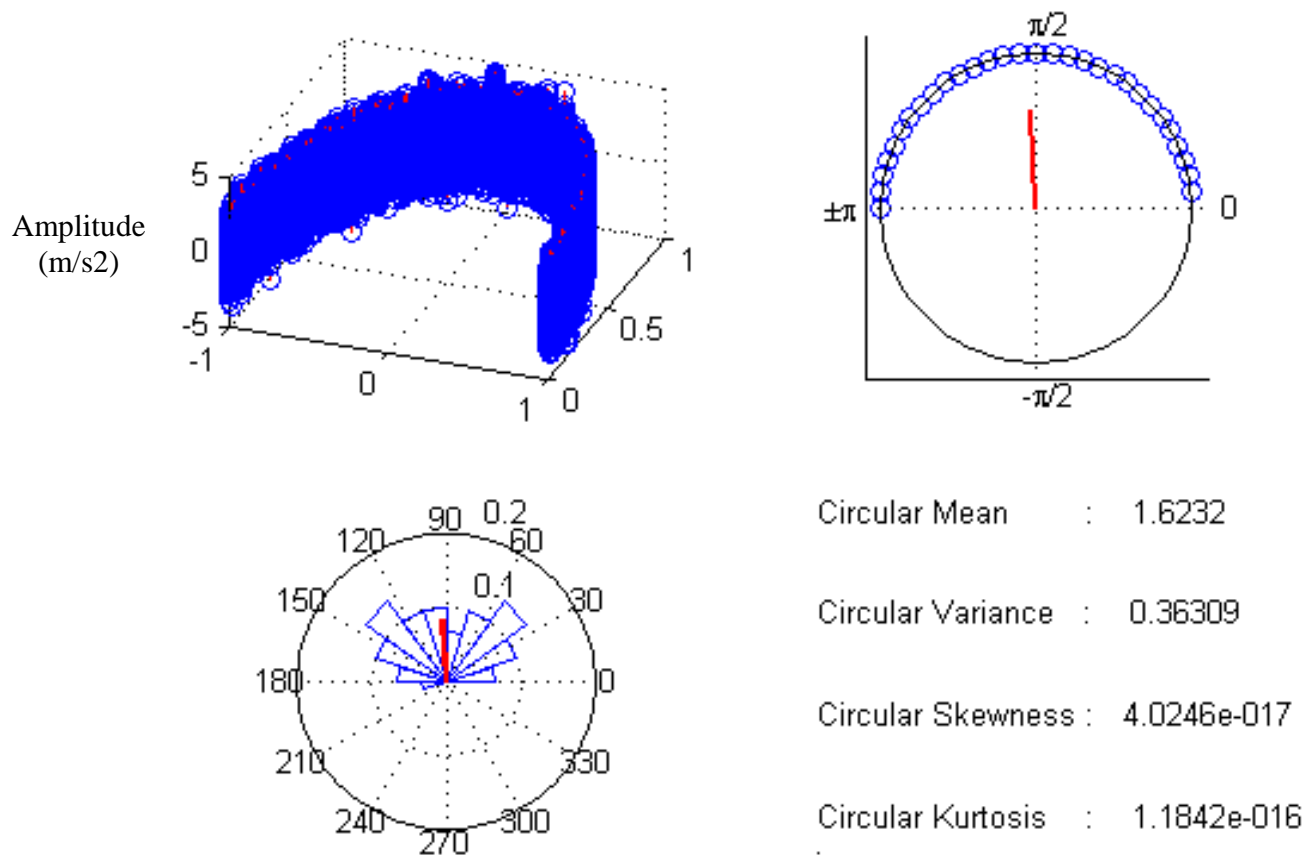

Fig. 15 Lab slewing bearing data acquired on March 1 (day 9th): (a) Vibration data in circular domain; (b) The 'occurrence' results plotted in circular domain (in radian); (c) Circular histogram (in degree); (d) Circular features calculation results.

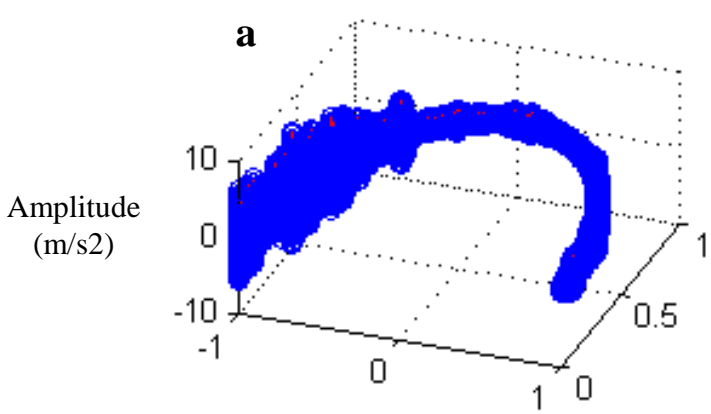

C

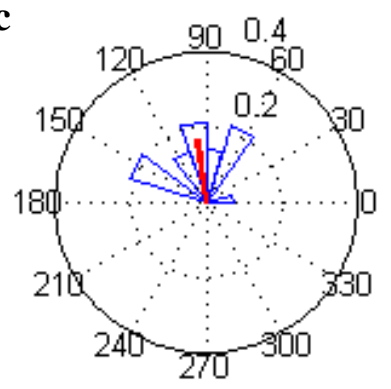

b

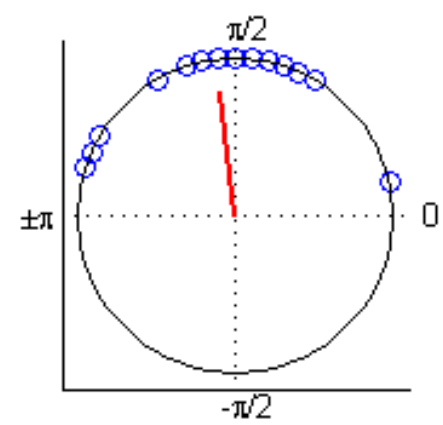

d

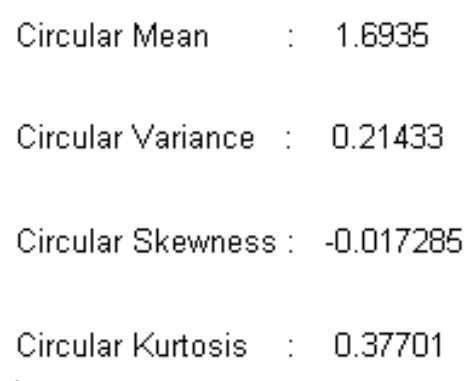

Fig. 16 Lab slewing bearing data acquired on May 25 (day 90 ${ }^{\text {th }}$ ): (a) Vibration data in circular domain; (b) The 'occurrence' results plotted in circular domain (in radian); (c) Circular histogram (in degree); (d) Circular features calculation results. 


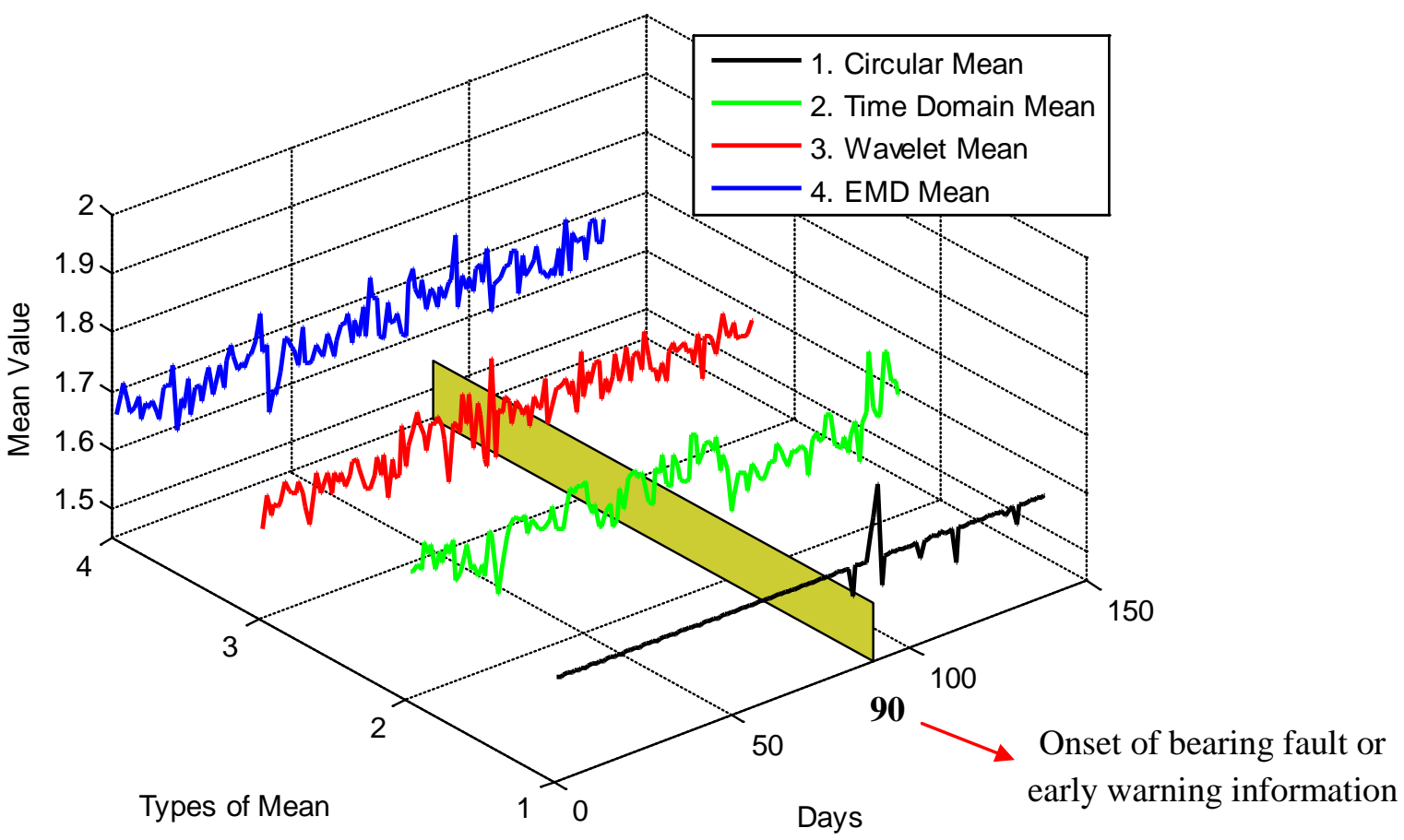

(a) Feature 1: Mean

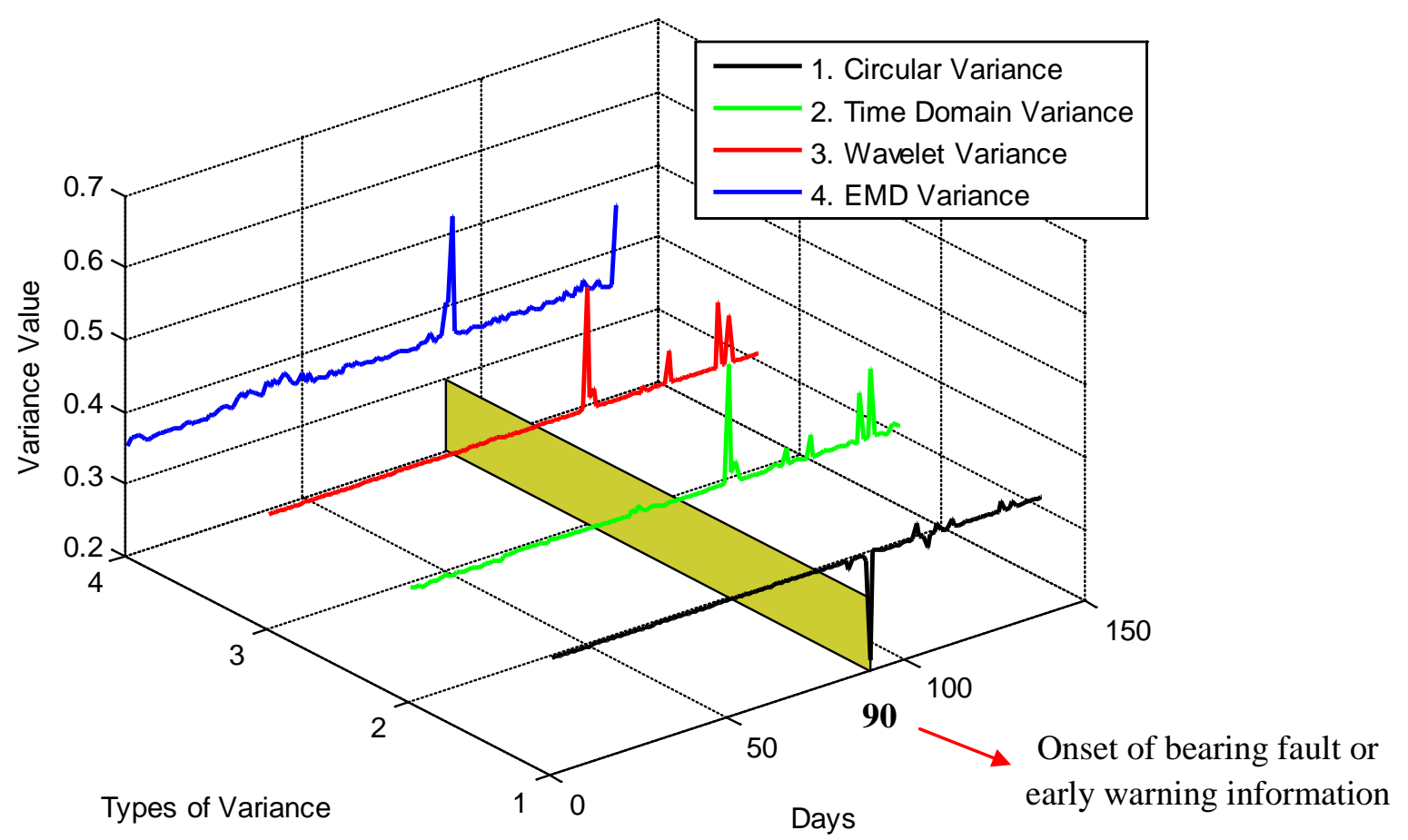

(b) Feature 2: Variance 


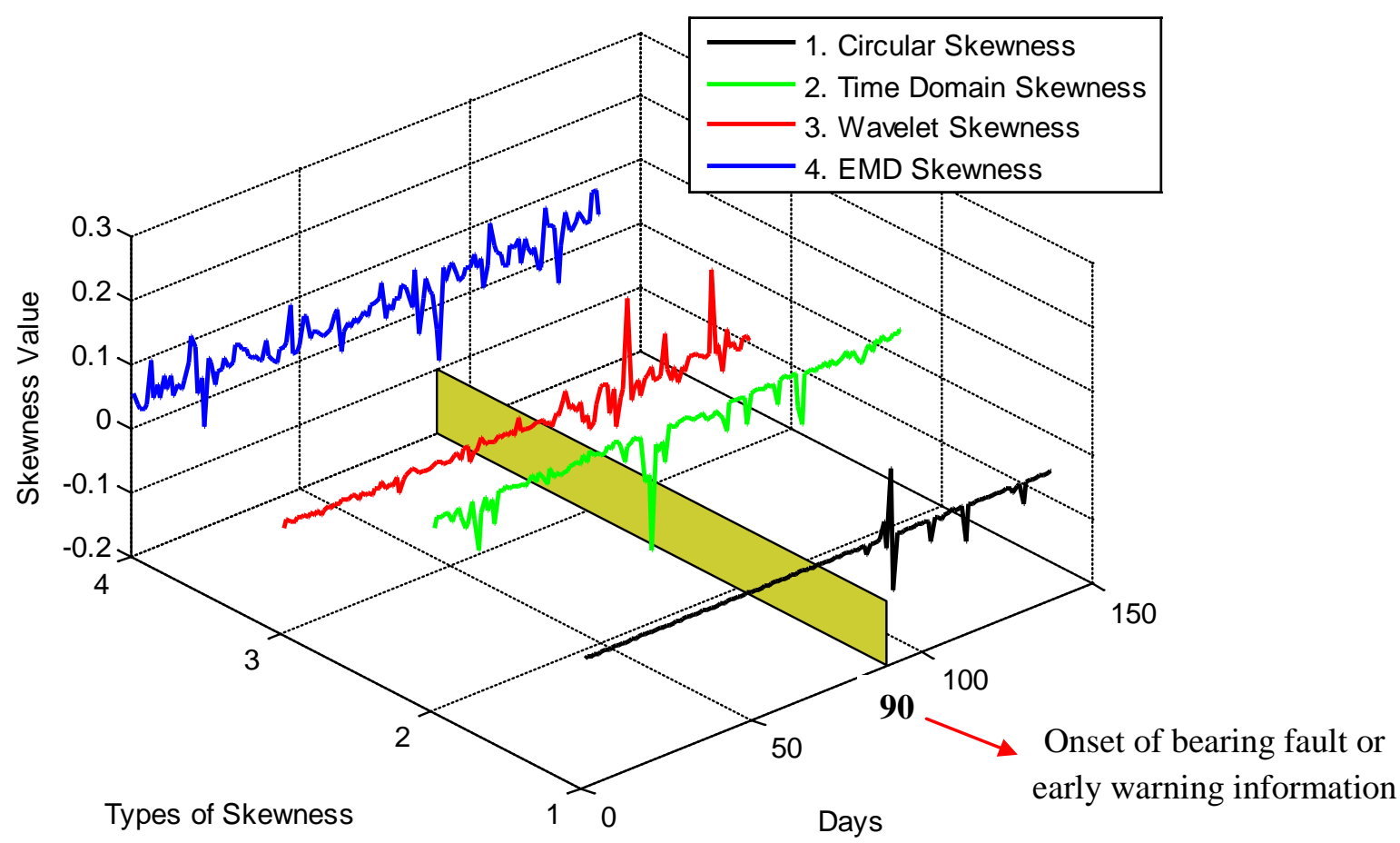

(c) Feature 3: Skewness

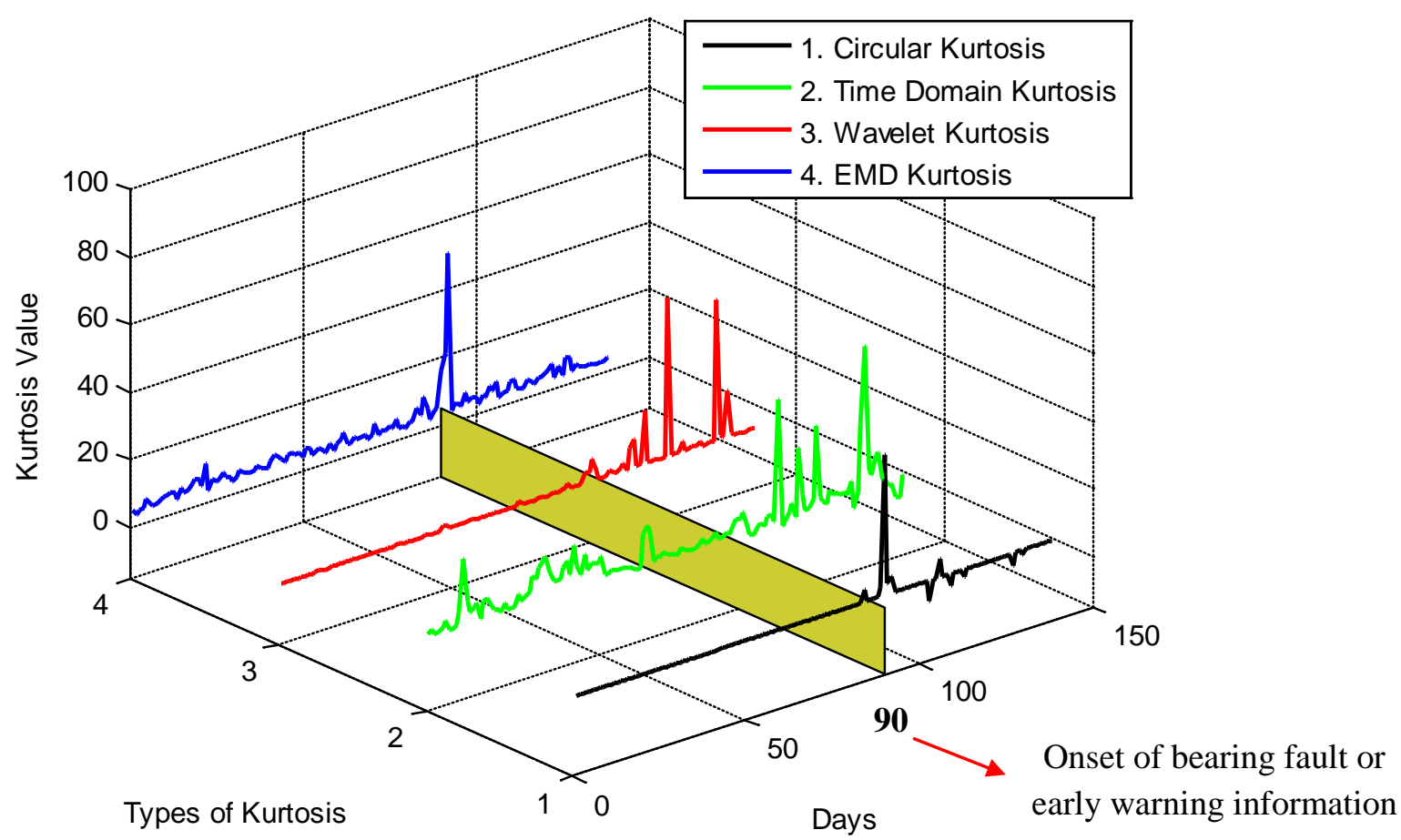

(d) Feature 4: Kurtosis 
Fig. 17 Circular-domain features extraction results and three comparable methods (time-domain features, wavelet decomposition and EMD) from February to August 2007 (138 days).

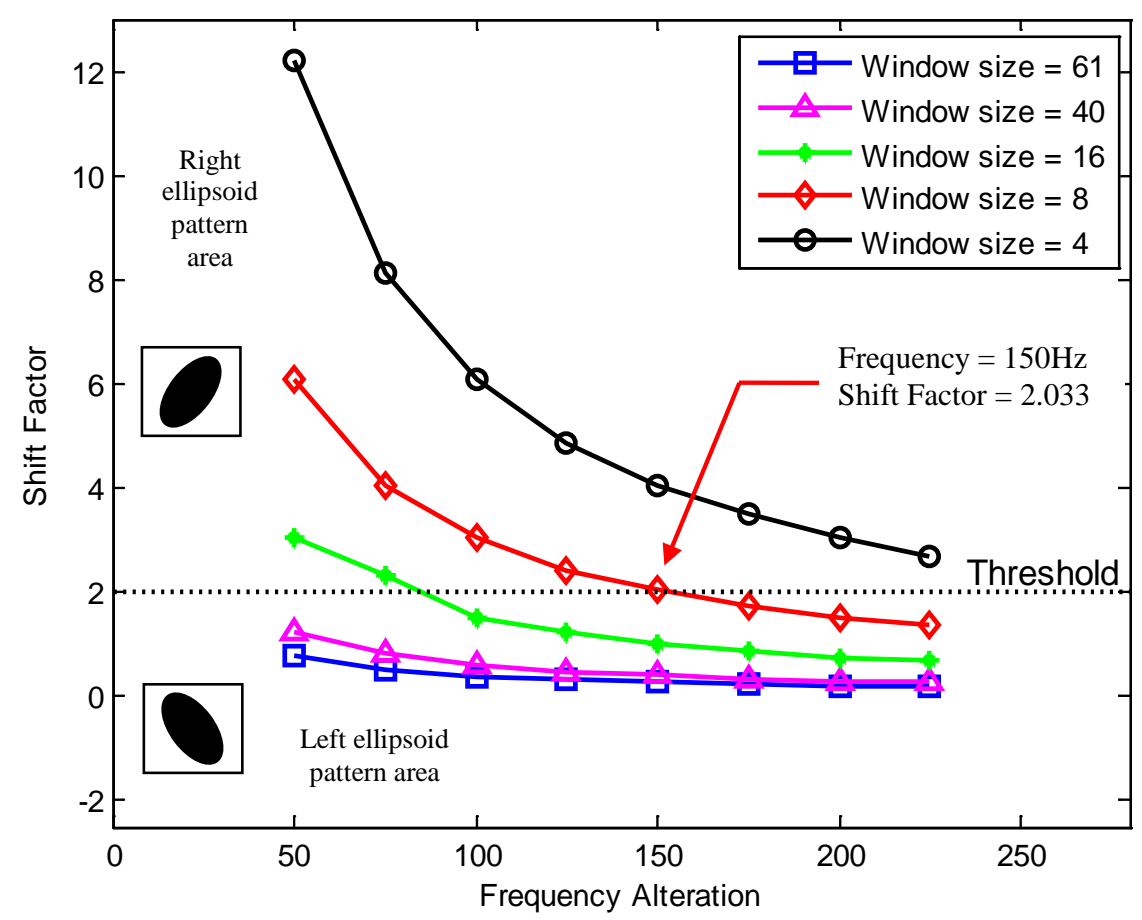

Fig. 18 Shift factor value for different frequency alteration and different window size.

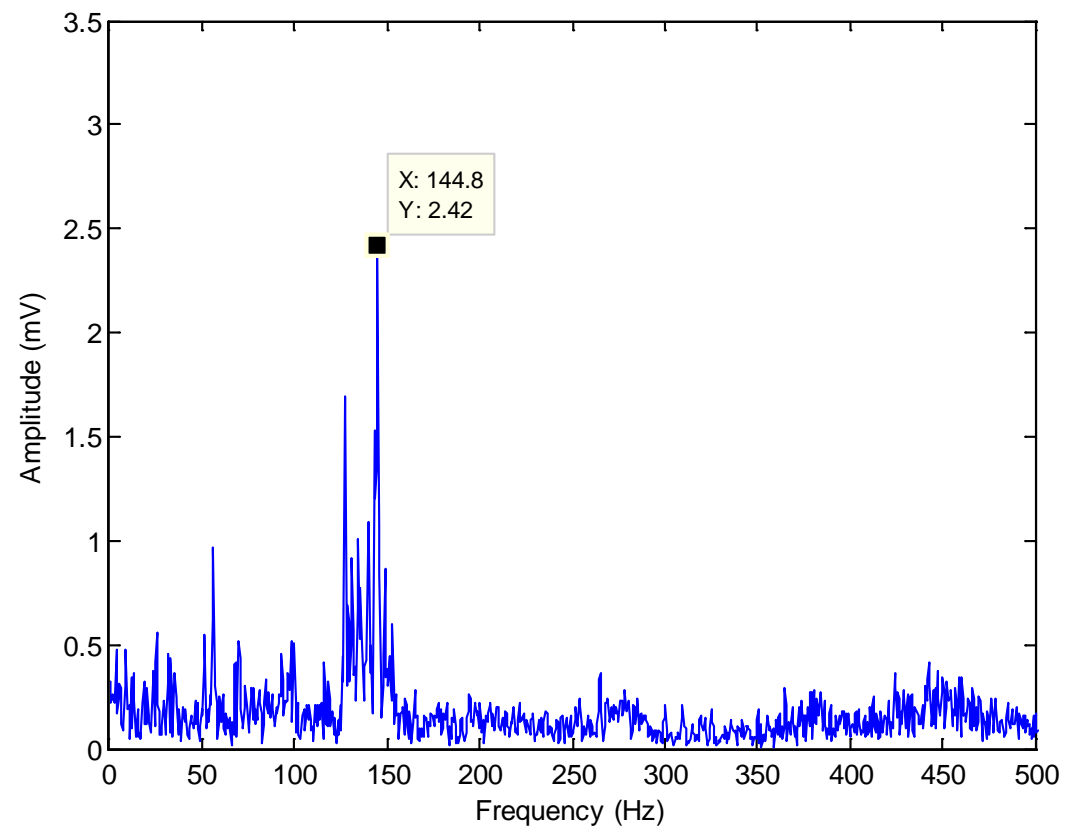

Fig. 19 FFT of slewing bearing data on May 25 data i.e. 90 days after the start [9]. 

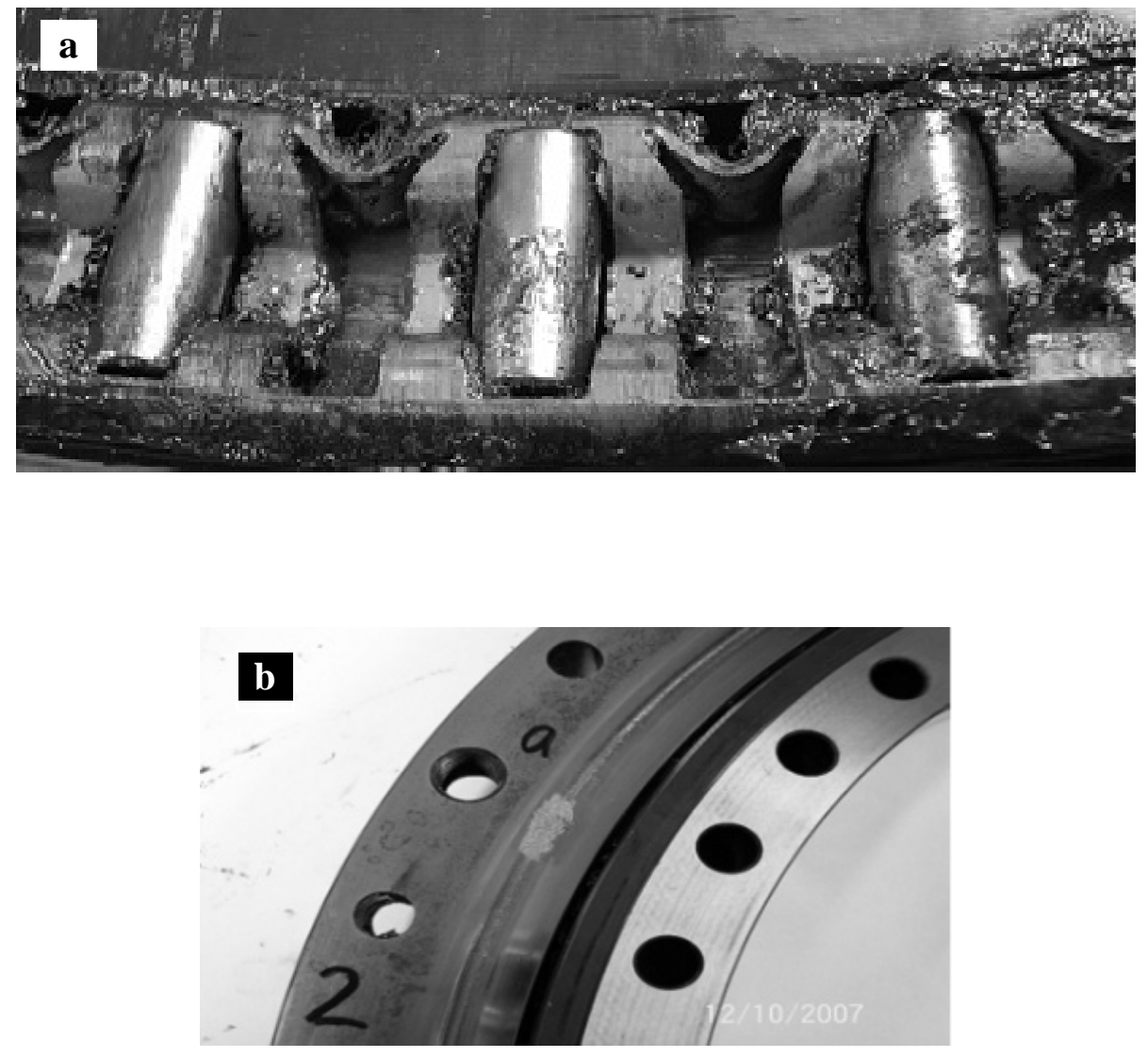

Fig. 20 (a) A view of damaged rollers in axial plane; (b) Outer raceway damage 